\title{
Soft-bottom macrobenthic faunal associations in the southern Chilean glacial fjord complex*
}

\author{
CARLOS RÍOS ${ }^{1}$, ERIKA MUTSCHKE ${ }^{2,3}$, AMÉRICO MONTIEL $^{2,3}$, DIETER GERDES $^{4}$ \\ and WOLF E. ARNTZ4 \\ ${ }^{1}$ Dirección de Programa Antártico, Universidad de Magallanes, P.O.Box 113-D, Punta Arenas, Chile. \\ E-mail: carlos.rios@umag.cl \\ ${ }^{2}$ Instituto de la Patagonia, Universidad de Magallanes, P.O.Box 113-D, Punta Arenas, Chile. \\ ${ }^{3}$ Centro de Estudios del Cuaternario de Fuego-Patagonia y Antártica Chilena (CEQUA), Punta Arenas, Chile. \\ ${ }^{4}$ Alfred Wegener Institute for Polar and Marine Research, P.O.Box 120161,D-27515, Bremerhaven, Germany.
}

SUMMARY: Macrobenthic associations were investigated at 29 sampling stations with a semi-quantitative Agassiz trawl, ranging from the South Patagonian Icefield to the Straits of Magellan in the South Chilean fjord system. A total of 1,895 individuals belonging to 131 species were collected. 19 species belong to colonial organisms, mainly Bryozoa (17 species) and Octocorallia ( 2 species). The phylum Echinodermata was the most diverse in species number (47 species), with asteroids ( 25 species) and ophiuroids (13 species) being the best represented within this taxon. Polychaeta was the second dominant group in terms of species richness (46 species). Multidimensional scaling ordination (MDS) separated two station groups, one related to fjords and channels off the South Patagonian Icefield and the second one to stations surrounding the Straits of Magellan. 45 species account for $90 \%$ of the dissimilarity between these two groups. These differences can mainly be explained by the influence of local environmental conditions determined by processes closely related to the presence/absence of glaciers. Abiotic parameters such as water depth, type of sediment and chemical features of the superficial sediment were not correlated with the numbers of individuals caught by the Agassiz trawl in each group of sampling stations.

Keywords: Magellan region, South Patagonian Icefield, Straits of Magellan, community structure, biodiversity.

RESUMEN: ASOCIACIONES FAUNísTICAS MACROBENTÓNICAS DE FONDOS BLANDOS EN EL COMPLEJO DE FIORDOS GLACIARES DE ChILE Austral. - Mediante el uso de una rastra Agassiz modificada se investigaron las asociaciones macrobentónicas presentes en 29 estaciones de muestreo, localizadas entre el Campo Patagónico de Hielo Sur y el Estrecho de Magallanes en el sistema de fiordos de Chile austral. Se colectó un total de 1.895 individuos pertenecientes a 131 especies, además de 19 especies de organismos coloniales, especialmente Bryozoa (17 especies) y Octocorallia (2 especies). El phylum Echinodermata fue el más diverso en términos de número de especies (47 especies), con una mejor representación de asteroideos (25 especies) y ofiuroideos (13 especies). El segundo grupo dominante en términos de riqueza de especies fue Polychaeta (46 especies). La técnica de "Escalamiento Multidimensional" (MDS) permitió separar dos grupos de estaciones: uno relacionado con los fiordos y canales del Campo Patagónico de Hielo Sur y el otro con las estaciones localizadas alrededor del Estrecho de Magallanes. Cuarenta y cinco especies explican el 90\% de disimilitud entre estos dos grupos. Las diferencias se pueden explicar principalmente por la influencia de condiciones ambientales locales, determinadas por procesos estrechamente relacionados con la presencia/ausencia de glaciares. No hubo correlación entre parámetros abióticos tales como profundidad, tipo de sedimentos y características químicas del sedimento superficial y el número de individuos capturados por la rastra Agassiz en cada grupo de estaciones de muestreo.

Palabras clave: Región de Magallanes, Campo de Hielo Patagónico Sur, estrecho de Magallanes, estructura comunitaria, biodiversidad. 


\section{INTRODUCTION}

Much of the Magellan region at the southern tip of South America is still under the influence of a massive, elongated, narrow ice sheet, which continues to be active but reached its largest extension during the Last Glacial Maximum period (around 19,000-23,000 yr ago; Hulton et al., 2002). At present, there is evidence of a wide and intensive retreat of several of the conforming fjordic glaciers which began 12,000 years ago (Moreno et al., 1999). The resulting geomorphologic area is a large complex of fjords and channels whose linear extension is estimated at about $30,000 \mathrm{~km}$ of linear shoreline.

In contrast to northern hemisphere fjord environments (e.g. Pearson, 1980; Gulliksen et al.,1985; Holte and Gulliksen, 1998; Larsen, 1997; Kendall et al., 2003; Wlodarska-Kowalczuk et al.,1998; Wlodarska-Kowalczuk and Pearson, 2004) and even in comparison with the Antarctic ecosystem (Dawber and Powell, 1997; Arntz et al.,1994; Jazdzewski et al., 2001), there is a lack of biological information on the Magellan region. However, this region represents a corner-stone for elucidating several biogeographical and ecological questions related to latitudinal gradients in species diversity or biomass (Clarke, 1992; Gray, 2001a; b; Piepenburg et al., 2002), origin and evolution of the (sub)Antarctic biota (Gallardo et al., 1992; Arntz and Ríos, 1999) or the split of zoogeographical units along the western coast of South America (Brattström and Johanssen, 1983; Lancellotti and Vásquez, 2000; Camus, 2001; Montiel et al., 2004).

The benthic sublittoral macrofauna inhabiting the Magellan region has been described mainly for the Straits of Magellan and related channels (e.g. Gutt et al., 1999; Gerdes and Montiel, 1999; Thatje and Mutschke, 1999; Brey and Gerdes, 1999; Montiel et al., 2001; Ríos et al., 2003) and mostly after the 1994 "Victor Hensen" scientific cruise in the Magellan region (Arntz and Gorny, 1996). Nevertheless, there still is a lack of comparative studies for the Magellan region, including an update of the taxonomic knowledge of this Subantarctic area (Arntz, 1999). A study on the benthic macrofauna along the entire Magellan coastal zone by Gerdes et $a l$. is still underway, focusing on the analysis of abundance and biomass of major taxa (e.g. Mollusca, Polychaeta, Crustacea and Echinodermata). The lowest values were obtained at the stations in the South Patagonian Icefield, with an increasing gradi- ent of abundance and biomass towards the southernmost sampling areas.

The present study encompasses a geographically wide range of the Magellan region and is based on data from semi-quantitative benthic research in this heterogeneous marine ecosystem. The aim is to give a better insight into the structure of benthic macrofaunal communities for the area at species level. Additionally, we identify and compare benthic key species, which are representative of the fjord and channel ecosystem in this region. Finally, we evaluate the influence of some physical environmental variables on the obtained macrobenthic distribution patterns.

\section{MATERIAL AND METHODS}

Samples were taken during the CIMAR-Fiordo III (October 1995) and CIMAR-Fiordo VII (November 1997) campaigns organised by the Chilean Comisión Oceanográfica Nacional (CONA) from on board the R/V "Vidal Gormaz". A total of 29 localities were sampled using a modified Agassiz trawl (AGT) $(3.15 \mathrm{~m}$ wide and $1.1 \mathrm{~m}$ high, with a mesh size of $10 \mathrm{~mm}$ ) at depths of 24-732 $\mathrm{m}$, ranging from the northern limit of the South Patagonian Icefield (SPI) to the eastern entrance of the Straits of Magellan (SM) (Fig. 1). Working time on the bottom for each haul averaged 12 min. Detailed sampling station data are summarised in Table 1.

Catch volumes of the AGT were relatively small at each station, in terms of both sediment and epibenthic fauna. Therefore, all the collected material was considered as a sample for later analysis and the number of caught specimens was used as representative of abundance for similarity analysis. The samples were immediately sorted onboard by separating all the collected fauna from the sediment using sieves with $1 \mathrm{~mm}$ mesh size. The sorted animals were preserved in a $5 \%$ formaldehyde seawater solution buffered with hexamethylentetramin.

The collected macrofauna was identified to the lowest possible taxonomic level, but for some taxa (e.g. Holothuroidea) identification was possible only to higher taxonomic levels. Organisms were identified based on standard publications (Retamal, 1974; Forcelli, 2000), experts who assisted in the identification of different taxa (see "Acknowledgement") and the identified benthic species at the "Edmundo Pisano Reference Collection" of the Instituto de la Patagonia, Universidad de Magallanes. 


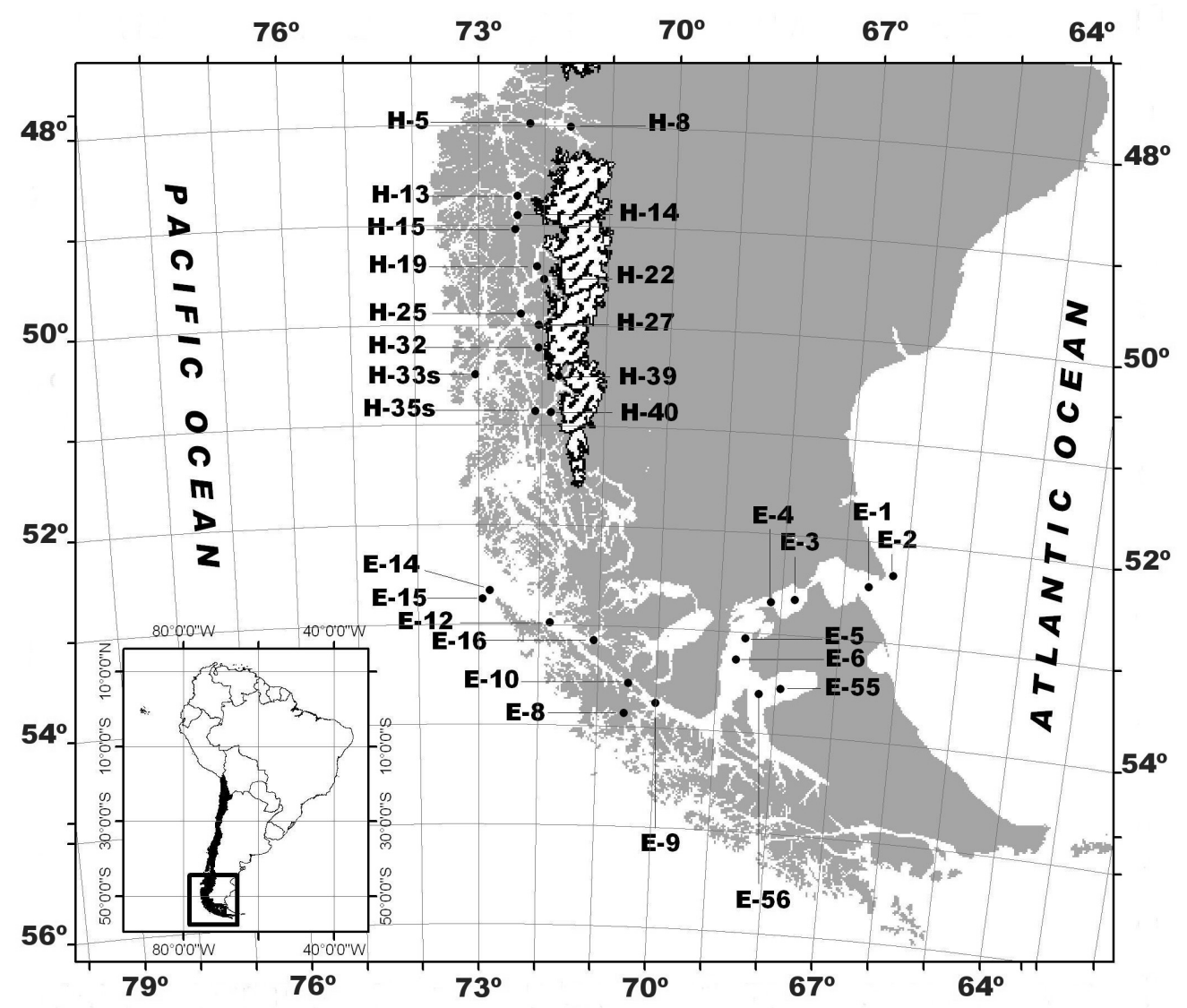

FIG. 1. - Map showing the Magellan region and the sampling sites in the South Patagonian Icefield (SPI) and Straits of Magellan (SM) areas.

TABLE 1. - List of stations sampled in the Magellan region. n.i. = no information.

\begin{tabular}{|c|c|c|c|c|c|c|c|c|c|}
\hline \multicolumn{2}{|c|}{ Station $N^{\circ}$ Locations } & \multirow{2}{*}{$\begin{array}{r}\text { Depth } \\
(\mathrm{m})\end{array}$} & \multirow{2}{*}{$\begin{array}{c}\text { Latitude S } \\
47^{\circ} 58.6\end{array}$} & \multirow{2}{*}{$\begin{array}{c}\text { Longitude W } \\
74^{\circ} 13.2\end{array}$} & \multirow{2}{*}{$\begin{array}{c}\begin{array}{c}\text { Sampling } \\
\text { date }\end{array} \\
22.08 .95\end{array}$} & \multirow{2}{*}{$\begin{array}{c}\begin{array}{c}\text { Bottom } \\
\text { type }\end{array} \\
\text { Mud }\end{array}$} & \multirow{2}{*}{$\begin{array}{c}\begin{array}{c}\mathrm{C} \text { org } \\
\text { (ug at/g) }\end{array} \\
540.7\end{array}$} & \multirow{2}{*}{$\begin{array}{c}\begin{array}{c}\mathrm{N} \text { org } \\
\text { (ug at/g) }\end{array} \\
86.5\end{array}$} & \multirow{2}{*}{$\begin{array}{c}\begin{array}{c}\mathrm{P} \text { total } \\
\text { (ug at/g) }\end{array} \\
14.4\end{array}$} \\
\hline H8 & Canal Baker & & & & & & & & \\
\hline H5 & Canal Baker & 382 & $48^{\circ} 00.3$ & $73^{\circ} 36.7$ & 22.08 .95 & Mud & 403.5 & 72.2 & 11.7 \\
\hline H13 & Canal Hammick & 345 & $48^{\circ} 42.3$ & $74^{\circ} 24.3$ & 23.08 .95 & Sand & 387.4 & 36.8 & 11.1 \\
\hline H14 & Angustura Inglesa & 385 & $46^{\circ} 53.9$ & $74^{\circ} 24.2$ & 23.08 .95 & Sand & 540.7 & 89.3 & 12.3 \\
\hline H15 & Paso del Indio & 166 & $49^{\circ} 02.4$ & $74^{\circ} 26.1$ & 23.08 .95 & Sand & 1024.9 & 187.8 & 16.2 \\
\hline H19 & Fiordo Eyre & 349 & $49^{\circ} 24.5$ & $74^{\circ} 05.9$ & 24.08 .95 & Mud & 212.1 & 30.7 & 11.4 \\
\hline $\mathrm{H} 22$ & Fiordo Falcon (sector Agüada) & 122 & $49^{\circ} 32.1$ & $73^{\circ} 59.1$ & 24.08 .95 & Mud & 355.1 & 58.8 & 9.4 \\
\hline $\mathrm{H} 27$ & Fiordo Penguin & 232 & $49^{\circ} 53.3$ & $74^{\circ} 20.3$ & 26.08 .95 & Mud & 581.1 & 80.5 & 8.5 \\
\hline $\mathrm{H} 25$ & Fiordo Penguin & 298 & $49^{\circ} 59.6$ & $74^{\circ} 03.7$ & 26.08 .95 & Mud & 322.8 & 48.1 & 11.8 \\
\hline H32 & Fiordo Europa & 66 & $50^{\circ} 13.5$ & $74^{\circ} 03.1$ & 27.08 .95 & Mud & 226.0 & 34.5 & 11.1 \\
\hline $\mathrm{H} 33 \mathrm{~S}$ & Canal Oeste & 75 & $50^{\circ} 29.6$ & $75^{\circ} 02.5$ & 28.08 .95 & Sand & n.i. & n.i. & n.i. \\
\hline $\mathrm{H} 35 \mathrm{~S}$ & Fiordo Peel (Paso la Piedra) & 117 & $50^{\circ} 51.7$ & $74^{\circ} 05.9$ & 29.08 .95 & Mud & 355.1 & 53.2 & 18.5 \\
\hline $\mathrm{H} 39$ & Fiordo Peel (Bahía Chubretovich) & 110 & $50^{\circ} 30.3$ & $73^{\circ} 44.3$ & 29.08 .95 & Mud & 266.3 & 23.0 & 10.7 \\
\hline $\mathrm{H} 40$ & Fiordo Amalia (Interior) & 148 & $50^{\circ} 52.3$ & $73^{\circ} 50.8$ & 29.08 .95 & Mud & 500.4 & 71.5 & 13.5 \\
\hline E1 & Boca Oriental E. de Magallanes & 70 & $52^{\circ} 27.4$ & $68^{\circ} 35.0$ & 06.10 .97 & Sand gravel & n.i. & n.i. & n.i. \\
\hline E2 & Bahía Posesión & 40 & $52^{\circ} 19.4$ & $69^{\circ} 12.2$ & 06.10 .97 & Mud & n.i. & n.i. & n.i. \\
\hline E3 & $1^{\circ}$ Angostura E. de Magallanes & 24 & $52^{\circ} 38.6$ & $69^{\circ} 46.48$ & 07.10 .97 & Sand gravel & n.i. & n.i. & n.i. \\
\hline E4 & $2^{\circ}$ Angostura E. de Magallanes & 41 & $52^{\circ} 41.1$ & $70^{\circ} 10.2$ & 07.10 .97 & Sand gravel & n.i. & n.i. & n.i. \\
\hline E5 & Paso Ancho & 88 & $53^{\circ} 03.6$ & $70^{\circ} 32.8$ & 07.10 .97 & Sand gravel & n.i. & n.i. & n.i. \\
\hline E6 & Paso Ancho & 177 & $53^{\circ} 16.5$ & $70^{\circ} 41.2$ & 07.10 .97 & Sand gravel & n.i. & n.i. & n.i. \\
\hline E56 & Bahía Inutil & 270 & $53^{\circ} 32.6$ & $69^{\circ} 54.7$ & 08.10 .97 & Mud & n.i. & n.i. & n.i. \\
\hline E55 & Bahía Inutil & 50 & $53^{\circ} 36.3$ & $70^{\circ} 16.0$ & 07.10 .97 & Mud & n.i. & n.i. & n.i. \\
\hline E8 & Bahía Snug & 260 & $53^{\circ} 51.8$ & $72^{\circ} 32.0$ & 14.10.97 & Mud & n.i. & n.i. & n.i. \\
\hline E9 & Isla Wood & 313 & $53^{\circ} 45.1$ & $71^{\circ} 59.9$ & 15.10 .97 & Mud & n.i. & n.i. & n.i. \\
\hline E10 & Paso Tortuoso & 404 & $53^{\circ} 33.7$ & $72^{\circ} 28.6$ & 15.10 .97 & Sand & n.i. & n.i. & n.i. \\
\hline E15 & Cabo Tamar & 604 & $52^{\circ} 58.6$ & $73^{\circ} 48.6$ & 16.10 .97 & Sand gravel & n.i. & n.i. & n.i. \\
\hline E14 & Boca Occidental E. de Magallanes & 66 & $52^{\circ} 39.5$ & $74^{\circ} 48.5$ & 16.10 .97 & Sand gravel & n.i. & n.i. & n.i. \\
\hline E12 & Boca Occidental E. de Magallanes & 90 & $52^{\circ} 44.4$ & $74^{\circ} 55.6$ & 16.10 .97 & Sand & n.i. & n.i. & n.i. \\
\hline E16 & Golfo Xaltegua & 411 & $53^{\circ} 8.65$ & $73^{\circ} 4.70$ & 16.10 .97 & Mud & n.i. & n.i. & n.i. \\
\hline
\end{tabular}


Data were analysed using the PRIMERv5 computer package (Clarke and Warwick, 1994). To analyse the spatial distribution of sampling sites over the study area, the MDS ("multidimensional scaling") program based on presence-absence matrix data was employed. This procedure also made it possible to consider colonial organisms (e.g. Gorgonaria and Bryozoa), which were collected at several sampling stations. Routine SIMPER ("similarity percentage") was used to discriminate species and their percentage of contribution to (dis) similarities within and between groups defined by the MDS. In this case, only solitary (counted) organisms were considered.

To determine whether the benthic distribution patterns showed relationships to environmental abiotic parameters, the BIO-ENV procedure from PRIMER was applied to data available for the study area. BIOENV (Clarke and Gorley, 2001) selects a combination of environmental factors best explaining a community pattern by maximising a Spearman rank correlation between the respective similarity matrices using all possible permutations of environmental factors. The similarity measure used was the Normalized Euclidean Distance. Environmental variables (water depth, substrate) and some sediment chemical features of the SPI (organic carbon and nitrogen, total phosphate) were obtained from Ahumada et al. (1996) and Silva and Prego (2002). Comparable information for the SM area is relatively scarce. The effects of water depth, bottom type, hydrodynamic regime and suspended particulate matter (Brambatti et al., 1991; Fontolan and Panella, 1991) were also analysed.

\section{RESULTS}

\section{Species composition and numerical dominance}

The sampling stations showed relatively low numbers of specimens in the catches by the Agassiz trawl. A total of 1,895 individuals were caught belonging to 5 epi- and endobenthos major taxa, from which 131 species were identified (Table 2). Another 19 species belonged to colonial taxa, mainly Bryozoa (17 species) and Octocorallia (2 species).

The taxon Echinodermata was the most diverse in numbers of species (47 species), with 25 species belonging to asteroids, 13 species to ophiuroids, 6 species to holothuroids and 3 to echinoids. Polychaeta (46 species) were the second dominant taxon in terms of species richness. Mollusca (25 species) were the third important taxon, encompassing Bivalvia (13 species), Gastropoda (8 species), Polyplacophora and Scaphopoda (2 species each).

Echinodermata were the taxon with the highest number of individuals, representing $51 \%$ of the total catch. Ophiuroidea were the most abundant class, representing $20 \%$ of the total collected organisms.

The frequency of occurrence of species at the sampling stations was low, in accordance with the number of macrofauna caught. The echinoid Pseudechinus magellanicus was caught at $35 \%$ of the sampling sites, whereas Ctenodiscus procurator (Asteroidea), Ophiuroglypha lymani (Ophiuroidea) and Euripodius latreillei (Crustacea) were collected at $24 \%$ of the stations.

\section{Comparison between sampling sites}

Two major groups of stations can be discriminated in the two-dimensional MDS configuration of the 29 sampling sites, based on presence-absence data (Fig 2a). The stress value for the ordination ( $\mathrm{s}=$ 0.13 ) corresponds to a good representation (Clarke and Warwick, 1994). One group includes all the stations associated with the South Patagonian Icefield and the second one comprises stations mostly situated in the Straits of Magellan area. The average dissimilarity of all pairwise coefficients in the SPI and SM group was 97.4. Of these, 10.5 were contributed by Ctenodiscus procurator, 7.3 by Ophiuroglypha lymani and 6.5 by Magellania venosa, accounting for $24.9 \%$ of the overall value of 97.4 (Table 3). These three species are representative of the SM station group. A total of 45 species account for $90 \%$ of the dissimilarity between these two groups. The stations in the Straits of Magellan show consistently higher catch levels than the stations off the SPI.

The ordination of sampling stations for the Straits of Magellan suggests a subordinate segregation of sampling sites, which was analysed in more detail considering only sample sites of this area (Fig 2b). In this case, distinct clusters of sites emerge, showing a relation to geographical positions across the Straits of Magellan. One group is related to the eastern entrance of the Straits (Boca Oriental, Primera Angostura, Segunda Angostura and Bahía Posesión), whereas stations from the Pacific entrance (e.g. Boca Occidental) form another separate subgroup. From sampling stations between the two Strait entrances (Atlantic and Pacific) there emerge two other subgroups. The sampling sites 
TABLE 2. - A list of marine invertebrate species found in the areas of study. Number of individuals caught with the Agassiz trawl at each station is included $(\mathrm{H}=$ South Patagonian Icefield stations; $\mathrm{E}=$ Straits of Magellan stations). Colonial organisms are indicated by $\mathrm{p}=$ presence.

Species/stations number $\quad$ H5 H8 H13H14 H15 H19 H22 H25 H27 H32H33sH35s H39 H40 E1 E2 E3 E4 E5 E6 E55E56 E8 E9 E10 E12 E14 E15E16

\section{Anthozoa}

$\begin{array}{lllllllllllllllllllllllllllllllllllll}\text { Thouarella variabilis } & 0 & 0 & 0 & 0 & 0 & 0 & 0 & 0 & 0 & 0 & 0 & 0 & 0 & 0 & 0 & 0 & 0 & 0 & 0 & \mathrm{p} & 0 & 0 & 0 & \mathrm{p} & 0 & 0 & 0 & 0 & 0 & 0\end{array}$ $\begin{array}{lllllllllllllllllllllllllllllllllll}\text { Primnoella sp } & 0 & 0 & 0 & 0 & 0 & 0 & 0 & 0 & 0 & 0 & 0 & 0 & 0 & 0 & 0 & 0 & 0 & 0 & 0 & 0 & 0 & 0 & 0 & \mathrm{p} & 0 & 0 & 0 & 0 & 0\end{array}$

\section{Polychaeta}

Terebellidae INDET

Polynoidea INDET

Opheliidae INDET

Ampharetidae INDET

Sabellidae INDET

Sabellariidae INDET

Lumbrineridae INDET

Eunereis patagonica

$\begin{array}{lll}0 & 0 & 0\end{array}$

$\begin{array}{llllllllllllllllllllllllllllll}0 & 0 & 0 & 0 & 0 & 0 & 0 & 0 & 0 & 0 & 0 & 0 & 0 & 0 & 0 & 0 & 0 & 2 & 0 & 0 & 0 & 0 & 0 & 0 & 0 & 0 & 0 & 0 & 0\end{array}$ $\begin{array}{llllllllllllllllllllllllllllllllllllllll}0 & 0 & 0 & 0 & 0 & 0 & 0 & 0 & 0 & 0 & 0 & 0 & 0 & 0 & 0 & 0 & 3 & 0 & 3 & 1 & 0 & 0 & 0 & 0 & 0 & 0 & 0 & 1 & 0\end{array}$ $\begin{array}{llllllllllllllllllllllllllllllllllllllllll}0 & 0 & 0 & 0 & 0 & 0 & 0 & 0 & 0 & 0 & 0 & 0 & 0 & 0 & 0 & 0 & 0 & 0 & 0 & 0 & 0 & 0 & 0 & 0 & 0 & 0 & 0 & 1 & 0\end{array}$ $\begin{array}{llllllllllllllllllllllllllllllllllllllll}0 & 0 & 0 & 0 & 0 & 0 & 0 & 0 & 0 & 0 & 0 & 0 & 0 & 0 & 0 & 0 & 0 & 0 & 0 & 1 & 0 & 0 & 0 & 0 & 0 & 0 & 0 & 0 & 0\end{array}$ $\begin{array}{lllllllllllllllllllllllllllllllllllll}0 & 0 & 0 & 0 & 0 & 0 & 0 & 0 & 0 & 0 & 0 & 0 & 0 & 0 & 3 & 1 & 0 & 1 & 0 & 0 & 0 & 0 & 0 & 0 & 0 & 0 & 0 & 0 & 0\end{array}$ $\begin{array}{llllllllllllllllllllllllllllllllllllll}0 & 0 & 0 & 0 & 0 & 0 & 0 & 0 & 0 & 0 & 0 & 0 & 0 & 0 & 0 & 0 & 0 & 0 & 0 & 1 & 0 & 0 & 0 & 0 & 0 & 0 & 0 & 0 & 0\end{array}$ $\begin{array}{lllllllllllllllllllllllllllllllllllllll}0 & 0 & 0 & 0 & 0 & 0 & 0 & 0 & 0 & 0 & 0 & 0 & 0 & 0 & 0 & 0 & 0 & 0 & 0 & 0 & 0 & 0 & 0 & 0 & 0 & 1 & 0 & 0 & 0\end{array}$ Platynereis sp. Onuphis pseudoirid $\begin{array}{llllllllllllllllllllllllllllllllllllllllll}0 & 0 & 0 & 0 & 0 & 0 & 0 & 0 & 0 & 0 & 0 & 0 & 0 & 0 & 0 & 0 & 0 & 0 & 2 & 0 & 0 & 0 & 0 & 0 & 0 & 0 & 0 & 0 & 0\end{array}$ Chaetopterus sp. Maldane sarsi

Melinna cristata

Nephtys paradoxa

Cirratulus sp.

Eunice pennata

Aphrodita magellanica

Abyssoninoe abyssorum Lumbrineris cingulata

Ninoe leptognatha

Asychis sp.

Nepthys sp.

Gymnonereis

hartmannschroederae Nicon maculata

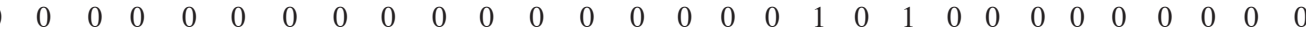
$\begin{array}{lllllllllllllllllllllllllllllllllllllll}0 & 0 & 0 & 0 & 0 & 0 & 0 & 0 & 0 & 0 & 0 & 0 & 0 & 0 & 0 & 0 & 0 & 0 & 0 & 0 & 0 & 0 & 0 & 0 & 0 & 0 & 0 & 11 & 0\end{array}$ $\begin{array}{lllllllllllllllllllllllllllllllllllllllll}0 & 1 & 3 & 0 & 1 & 0 & 0 & 0 & 0 & 1 & 0 & 0 & 0 & 0 & 0 & 0 & 0 & 0 & 0 & 0 & 0 & 0 & 0 & 0 & 0 & 0 & 0 & 0 & 0\end{array}$ $\begin{array}{llllllllllllllllllllllllllllllllllllllll}0 & 0 & 1 & 0 & 0 & 0 & 0 & 0 & 0 & 0 & 0 & 0 & 0 & 0 & 0 & 0 & 0 & 0 & 0 & 0 & 0 & 0 & 0 & 0 & 0 & 0 & 0 & 0 & 0\end{array}$ $\begin{array}{lllllllllllllllllllllllllllllllllllllllll}0 & 0 & 0 & 0 & 1 & 0 & 0 & 0 & 1 & 0 & 0 & 1 & 0 & 0 & 0 & 0 & 0 & 0 & 0 & 0 & 0 & 0 & 0 & 0 & 0 & 0 & 0 & 0 & 0\end{array}$

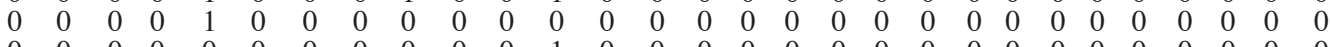
$\begin{array}{lllllllllllllllllllllllllllllllllllllll}0 & 0 & 0 & 0 & 0 & 0 & 0 & 0 & 0 & 0 & 0 & 1 & 0 & 0 & 0 & 0 & 0 & 0 & 0 & 0 & 0 & 0 & 0 & 0 & 0 & 0 & 0 & 0 & 0\end{array}$ $\begin{array}{llllllllllllllllllllllllllllllllllllllll}0 & 0 & 1 & 0 & 0 & 0 & 0 & 1 & 0 & 0 & 0 & 0 & 0 & 0 & 0 & 0 & 0 & 0 & 0 & 0 & 0 & 0 & 0 & 0 & 0 & 0 & 0 & 0 & 0\end{array}$ $\begin{array}{llllllllllllllllllllllllllllllll}0 & 0 & 0 & 0 & 1 & 0 & 0 & 0 & 1 & 0 & 0 & 0 & 0 & 4 & 0 & 0 & 0 & 0 & 0 & 0 & 0 & 0 & 0 & 0 & 0 & 0 & 0 & 0 & 0\end{array}$

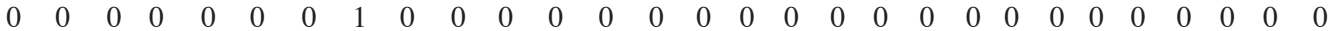
$\begin{array}{llllllllllllllllllllllllllllllllllllll}0 & 0 & 0 & 0 & 0 & 2 & 0 & 0 & 0 & 0 & 0 & 0 & 0 & 0 & 0 & 0 & 0 & 0 & 0 & 0 & 0 & 0 & 0 & 0 & 0 & 0 & 0 & 0 & 0\end{array}$

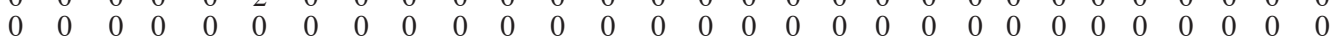
$\begin{array}{lllllllllllllllllllllllllllllllllllllllllll}0 & 0 & 0 & 0 & 0 & 0 & 0 & 3 & 0 & 0 & 0 & 0 & 0 & 0 & 0 & 0 & 0 & 0 & 0 & 0 & 0 & 0 & 0 & 0 & 0 & 0 & 0 & 0 & 0\end{array}$

Neanthes $c f$. abyssorum Neanthes kerguelensis

Travisia kerguelensis $\begin{array}{lllllllllllllllllllllllllllllllllllllll}\text { Anaitides sp. } & 0 & 0 & 0 & 0 & 0 & 0 & 0 & 0 & 0 & 0 & 1 & 0 & 0 & 0 & 0 & 0 & 0 & 0 & 0 & 0 & 0 & 0 & 0 & 0 & 0 & 0 & 0 & 0 & 0 \\ \text { Harmothoe cf. xanthena } & 0 & 0 & 0 & 0 & 0 & 0 & 0 & 0 & 0 & 0 & 1 & 0 & 0 & 0 & 0 & 0 & 0 & 0 & 0 & 0 & 0 & 0 & 0 & 0 & 0 & 0 & 0 & 0 & 0\end{array}$

$\begin{array}{lllllllllllllllllllllllllllllllllll}0 & 1 & 0 & 0 & 0 & 0 & 0 & 0 & 0 & 0 & 0 & 0 & 0 & 0 & 0 & 0 & 0 & 0 & 0 & 0 & 0 & 0 & 0 & 0 & 0 & 0 & 0 & 0 & 0\end{array}$

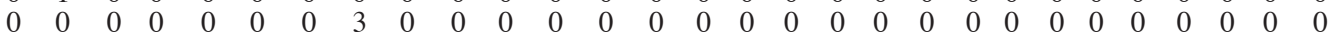
$\begin{array}{llllllllllllllllllllllllllllll}0 & 0 & 0 & 0 & 1 & 0 & 0 & 0 & 0 & 0 & 0 & 0 & 0 & 0 & 0 & 0 & 0 & 0 & 0 & 0 & 0 & 0 & 0 & 0 & 0 & 0 & 0 & 0 & 0\end{array}$

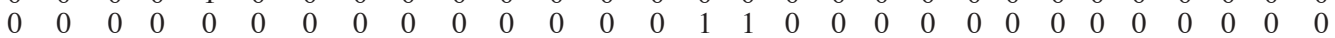
$\begin{array}{llllllllllllllllllllllllllllllllllllllllll}0 & 0 & 0 & 0 & 0 & 0 & 0 & 0 & 1 & 0 & 0 & 0 & 0 & 0 & 0 & 0 & 0 & 0 & 0 & 0 & 0 & 0 & 0 & 0 & 0 & 0 & 0 & 0 & 0\end{array}$

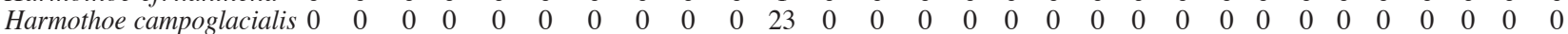
Hololepida sp. Polyeunoa laevis $\begin{array}{lllllllllllllllllllllllllllllllllllllllll}0 & 0 & 0 & 0 & 0 & 0 & 0 & 0 & 0 & 0 & 1 & 0 & 0 & 0 & 0 & 0 & 0 & 0 & 0 & 0 & 0 & 0 & 0 & 0 & 0 & 0 & 0 & 0 & 0\end{array}$

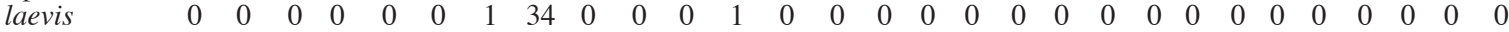

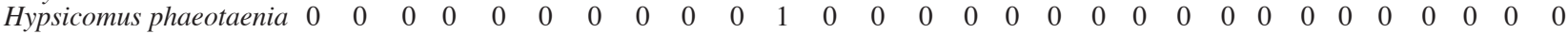
$\begin{array}{llllllllllllllllllllllllllllllllllll}\text { Leanira quatrefagesi } & 0 & 0 & 0 & 0 & 6 & 3 & 0 & 1 & 2 & 0 & 3 & 0 & 2 & 2 & 0 & 0 & 0 & 0 & 0 & 0 & 0 & 0 & 0 & 0 & 0 & 0 & 0 & 0 & 0\end{array}$

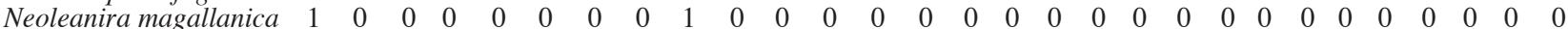
Laonice sp.

Sternaspis scutata

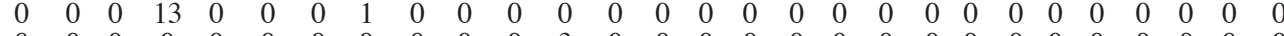
$\begin{array}{lllllllllllllllllllllllllllllllllll}\text { Eteone sculpta } & 0 & 0 & 0 & 0 & 0 & 0 & 0 & 0 & 0 & 0 & 0 & 0 & 0 & 0 & 1 & 0 & 0 & 0 & 0 & 0 & 0 & 0 & 0 & 0 & 0 & 0 & 0 & 0 & 0 & 0\end{array}$ $\begin{array}{llllllllllllllllllllllllllllllllll}\text { Sthenolepis magellanica } & 0 & 0 & 0 & 0 & 0 & 0 & 0 & 0 & 0 & 0 & 0 & 0 & 0 & 0 & 0 & 0 & 0 & 0 & 0 & 0 & 0 & 0 & 0 & 0 & 1 & 0 & 0 & 0 & 0\end{array}$ $\begin{array}{llllllllllllllllllllllllllllllllllllll}\text { Nothria sp. } & 0 & 0 & 0 & 0 & 0 & 0 & 0 & 0 & 0 & 0 & 0 & 0 & 0 & 0 & 0 & 0 & 0 & 0 & 0 & 0 & 0 & 0 & 0 & 0 & 1 & 0 & 0 & 0 & 0 & 0\end{array}$ $\begin{array}{llllllllllllllllllllllllllllllll}\text { Lanice cf. flabellum } & 0 & 0 & 0 & 0 & 0 & 0 & 0 & 0 & 0 & 0 & 1 & 0 & 0 & 0 & 0 & 0 & 0 & 0 & 0 & 0 & 0 & 0 & 0 & 0 & 0 & 0 & 0 & 0 & 0\end{array}$ $\begin{array}{llllllllllllllllllllllllllllllllllll}\text { Nicolea chilensis } & 0 & 0 & 0 & 0 & 0 & 0 & 0 & 0 & 0 & 0 & 7 & 0 & 0 & 0 & 0 & 0 & 0 & 0 & 0 & 0 & 0 & 0 & 0 & 0 & 0 & 0 & 0 & 0 & 0\end{array}$ $\begin{array}{llllllllllllllllllllllllllllllllll}\text { Pista cristata } & 0 & 0 & 7 & 0 & 0 & 0 & 0 & 1 & 0 & 0 & 0 & 0 & 0 & 0 & 0 & 0 & 0 & 0 & 0 & 0 & 0 & 0 & 0 & 0 & 0 & 0 & 0 & 0 & 0 & 0\end{array}$

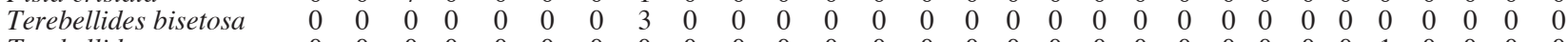
$\begin{array}{llllllllllllllllllllllllllllllllllll}\text { Terebellides sp. } & 0 & 0 & 0 & 0 & 0 & 0 & 0 & 0 & 0 & 0 & 0 & 0 & 0 & 0 & 0 & 0 & 0 & 0 & 0 & 0 & 0 & 0 & 0 & 0 & 1 & 0 & 0 & 0 & 0\end{array}$

\section{Decapoda}

$\begin{array}{lllllllllllllllllllllllllllllllllll}\text { Eurypodius latreillei } & 0 & 0 & 0 & 0 & 0 & 0 & 0 & 0 & 0 & 0 & 8 & 1 & 0 & 0 & 0 & 0 & 0 & 0 & 2 & 2 & 0 & 0 & 0 & 0 & 1 & 0 & 2 & 4 & 0\end{array}$

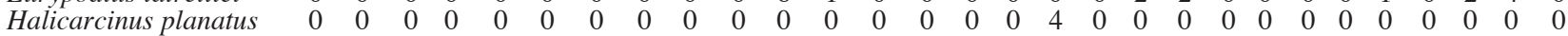
$\begin{array}{lllllllllllllllllllllllllllllllllll}\text { Stereomastis suhmi } & 1 & 0 & 0 & 0 & 0 & 0 & 0 & 0 & 0 & 0 & 0 & 0 & 0 & 0 & 0 & 0 & 0 & 0 & 0 & 0 & 0 & 0 & 0 & 0 & 0 & 0 & 0 & 0 & 0 & 0\end{array}$

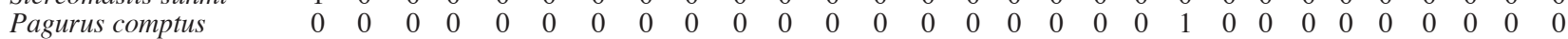

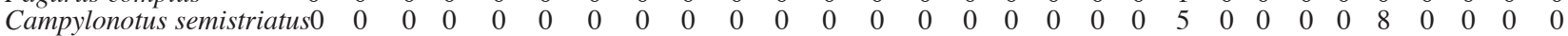
Libidoclaea smithi $\quad \begin{array}{llllllllllllllllllllllllllllllllllllllllllll}0 & 0 & 1 & 3 & 0 & 0 & 0 & 1 & 0 & 0 & 0 & 0 & 0 & 0 & 0 & 0 & 0 & 0 & 0 & 0 & 0 & 0 & 0 & 0 & 2 & 0 & 0 & 0 & 0\end{array}$ $\begin{array}{llllllllllllllllllllllllllllllllllll}\text { Munida subrugosa } & 0 & 0 & 0 & 0 & 0 & 1 & 0 & 0 & 0 & 0 & 0 & 0 & 2 & 0 & 0 & 0 & 0 & 0 & 0 & 0 & 0 & 0 & 2 & 0 & 0 & 0 & 0 & 0 & 0\end{array}$ $\begin{array}{lllllllllllllllllllllllllllllllllll}\text { Peltarion spinosulum } & 0 & 0 & 0 & 0 & 0 & 0 & 0 & 0 & 0 & 0 & 0 & 0 & 0 & 1 & 0 & 0 & 0 & 0 & 0 & 2 & 0 & 0 & 0 & 0 & 0 & 0 & 0 & 0 & 0\end{array}$

\section{Isopoda}

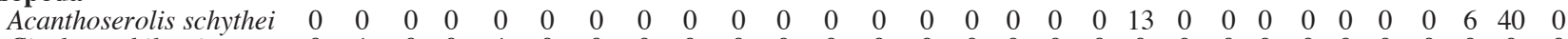
$\begin{array}{llllllllllllllllllllllllllllllllll}\text { Cirolana chilensis } & 0 & 1 & 0 & 0 & 1 & 0 & 0 & 0 & 0 & 0 & 0 & 0 & 0 & 0 & 0 & 0 & 0 & 0 & 0 & 0 & 0 & 0 & 0 & 0 & 0 & 0 & 0 & 0 & 0\end{array}$ Aega sp.

Thoracica

Austromegabalanus psittacus

Polyplacophora 
TABLE 2 (Cont.). - A list of marine invertebrate species found in the areas of study. Number of individuals caught with the Agassiz trawl at each station is included $(\mathrm{H}=$ South Patagonian Icefield stations; $\mathrm{E}=$ Straits of Magellan stations). Colonial organisms are indicated by $\mathrm{p}=\mathrm{presence}$.

Species/stations number

H5 H8 H13H14 H15 H19 H22 H25 H27 H32H33sH35s H39 H40 E1 E2 E3 E4 E5 E6 E55E56 E8 E9 E10 E12 E14 E15E16

\section{Gastropoda}

Crepidula dilatata

Trochita pileolus

Trochita pileus

Adelomelon ancilla

Berthella platei

Trophon geversianus

Pareuthria plumbea

Photinula caerulescens

\section{Bivalvia}

Lucinoma lamellata

Ennucula grayi

Acesta patagonica

Camptonectus (P.) subhyalinus

Limopsis marionensis

Limopsis sp.

Cyclocardia velutina

Tindaria virens

Nucula sp.

Chlamys patagonica

Yoldia cf. woodwardi

Yoldia eightsii

Scaphopoda

Dentalium majorinum

Dentalium cf. perceptum

Bryozoa

Reteporella magellensis

Nevianipora milneana

Hornera sp.

Microporella hyadesi

Rhabdopleura normani

Adeonella sp.

Orthoporidra petiolata

Aspidostoma giganteum

Catadysis pygmaeum

Carbacea ovoidea

Hippodinella adpressa

Cellaria malvinensis

Heteporella chilensis

Smittina lebruni

Ogivalia elegans

Arachnopusia monoceros

Fasciculipora meandrina

Articulata

Terebratella dorsata

Magellania venosa

Asteroidea

Ctenodiscus procurator

Asterina fimbriata

Cheiraster (Luidiaster)

planeta

Solaster regularis

Poraniopsis mira

Lophaster stellans

Doraster qawashqari

Cosmasterias lurida

Henricia obesa

Henricia studeri

Labidiaster radiosus

Calyptraster tenuissimus

Odontaster penicillatus

Odontaster meridionalis

Ceramaster patagonicus

Hippasterias sp.

Porania antarctica

magellanica

Anasterias antarctica

Anasterias sp.

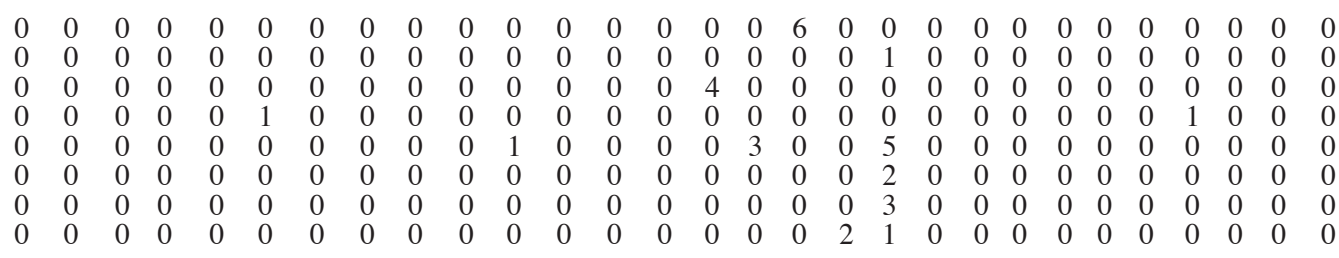

$\begin{array}{llllllllllllllllllllllllllllll}0 & 0 & 0 & 0 & 0 & 0 & 0 & 0 & 0 & 0 & 0 & 0 & 0 & 0 & 0 & 0 & 3 & 0 & 0 & 0 & 0 & 0 & 0 & 0 & 0 & 0 & 0 & 3 & 0\end{array}$ $\begin{array}{llllllllllllllllllllllllllllllllllllll}0 & 0 & 0 & 0 & 4 & 0 & 0 & 0 & 0 & 0 & 0 & 0 & 0 & 0 & 0 & 0 & 0 & 0 & 0 & 0 & 0 & 0 & 0 & 0 & 0 & 0 & 0 & 0 & 0\end{array}$ $\begin{array}{lllllllllllllllllllllllllllllllllllllllllll}1 & 0 & 0 & 0 & 0 & 0 & 0 & 0 & 0 & 0 & 0 & 0 & 0 & 8 & 0 & 0 & 0 & 0 & 6 & 0 & 0 & 0 & 0 & 0 & 0 & 0 & 0 & 0 & 0\end{array}$ $\begin{array}{llllllllllllllllllllllllllllll}0 & 0 & 0 & 0 & 0 & 0 & 0 & 0 & 0 & 0 & 0 & 0 & 0 & 0 & 0 & 0 & 0 & 0 & 1 & 0 & 0 & 0 & 0 & 0 & 0 & 0 & 0 & 0 & 0\end{array}$

$\begin{array}{llllllllllllllllllllllllllllllllll}0 & 0 & 0 & 0 & 0 & 0 & 0 & 191 & 0 & 0 & 0 & 0 & 0 & 0 & 0 & 0 & 0 & 0 & 0 & 0 & 0 & 0 & 0 & 0 & 0 & 0 & 0 & 0 & 0 \\ 0 & 0 & 0 & 1 & 0 & 0 & 0 & 0 & 1 & 0 & 0 & 1 & 0 & 0 & 0 & 0 & 0 & 0 & 0 & 72 & 0 & 0 & 5 & 0 & 0 & 0 & 0 & 0 & 0\end{array}$

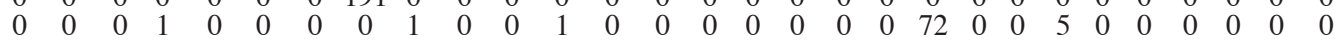
$\begin{array}{lllllllllllllllllllllllllllllllllllllllllll}0 & 0 & 0 & 1 & 0 & 0 & 0 & 0 & 0 & 0 & 0 & 0 & 0 & 0 & 0 & 0 & 0 & 0 & 0 & 0 & 0 & 0 & 0 & 0 & 0 & 0 & 0 & 0 & 0\end{array}$ $\begin{array}{lllllllllllllllllllllllllllllll}0 & 0 & 0 & 0 & 2 & 0 & 0 & 0 & 0 & 0 & 0 & 0 & 0 & 0 & 0 & 0 & 0 & 0 & 0 & 4 & 0 & 0 & 0 & 14 & 0 & 0 & 0 & 0 & 0\end{array}$ $\begin{array}{llllllllllllllllllllllllllllll}0 & 0 & 0 & 0 & 1 & 0 & 0 & 0 & 0 & 0 & 0 & 0 & 0 & 0 & 0 & 0 & 0 & 0 & 0 & 0 & 0 & 0 & 0 & 0 & 0 & 0 & 0 & 0 & 0\end{array}$ $\begin{array}{llllllllllllllllllllllllllllll}0 & 0 & 0 & 0 & 5 & 0 & 0 & 0 & 0 & 0 & 0 & 0 & 0 & 0 & 0 & 0 & 0 & 0 & 0 & 0 & 0 & 0 & 0 & 0 & 0 & 0 & 0 & 0 & 0 & 0\end{array}$

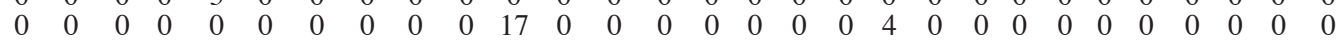
$\begin{array}{llllllllllllllllllllllllllllll}0 & 0 & 0 & 0 & 0 & 2 & 0 & 0 & 0 & 0 & 0 & 0 & 0 & 0 & 0 & 0 & 0 & 0 & 0 & 0 & 0 & 0 & 0 & 0 & 0 & 0 & 0 & 0 & 0\end{array}$ $\begin{array}{lllllllllllllllllllllllllllllllllllllllll}0 & 0 & 0 & 0 & 0 & 0 & 0 & 0 & 0 & 0 & 0 & 0 & 0 & 0 & 0 & 0 & 0 & 0 & 0 & 0 & 0 & 0 & 0 & 0 & 0 & 0 & 0 & 0 & 0\end{array}$

$\begin{array}{llllllllllllllllllllllllllllll}0 & 0 & 0 & 0 & 1 & 0 & 0 & 0 & 5 & 0 & 0 & 0 & 0 & 0 & 0 & 0 & 0 & 0 & 0 & 0 & 12 & 0 & 10 & 0 & 0 & 0 & 0 & 0 & 1\end{array}$ $\begin{array}{lllllllllllllllllllllllllllllllllllllllllllll}0 & 0 & 0 & 0 & 0 & 0 & 0 & 0 & 0 & 0 & \mathrm{p} & 0 & 0 & 0 & 0 & 0 & 0 & 0 & 0 & 0 & 0 & 0 & 0 & 0 & 0 & 0 & 0 & 0 & 0\end{array}$

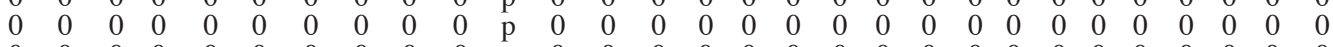
$\begin{array}{llllllllllllllllllllllllllllll}0 & 0 & 0 & 0 & 0 & 0 & 0 & 0 & 0 & 0 & \mathrm{p} & 0 & 0 & 0 & 0 & 0 & 0 & 0 & 0 & 0 & 0 & 0 & 0 & 0 & 0 & 0 & 0 & 0 & 0\end{array}$ $\begin{array}{lllllllllllllllllllllllllllllllllllllll}0 & 0 & 0 & 0 & 0 & 0 & 0 & 0 & 0 & 0 & \mathrm{p} & 0 & 0 & 0 & 0 & 0 & 0 & 0 & 0 & 0 & 0 & 0 & 0 & 0 & 0 & 0 & 0 & 0 & 0\end{array}$ $\begin{array}{llllllllllllllllllllllllllllll}0 & 0 & 0 & 0 & 0 & 0 & 0 & 0 & 0 & 0 & \mathrm{p} & 0 & 0 & 0 & 0 & 0 & 0 & 0 & 0 & 0 & 0 & 0 & 0 & 0 & 0 & 0 & 0 & 0 & 0\end{array}$ $\begin{array}{llllllllllllllllllllllllllllllll}0 & 0 & 0 & 0 & 0 & 0 & 0 & 0 & 0 & 0 & \mathrm{p} & 0 & 0 & 0 & 0 & 0 & 0 & 0 & 0 & 0 & 0 & 0 & 0 & \mathrm{p} & 0 & 0 & 0 & 0 & 0\end{array}$ $\begin{array}{llllllllllllllllllllllllllllllllllllll}0 & 0 & 0 & 0 & 0 & 0 & 0 & 0 & 0 & 0 & \mathrm{p} & 0 & 0 & 0 & 0 & 0 & 0 & 0 & 0 & 0 & 0 & 0 & 0 & 0 & 0 & 0 & 0 & 0 & 0\end{array}$ $\begin{array}{lllllllllllllllllllllllllllllllllllllllll}0 & 0 & 0 & 0 & 0 & 0 & 0 & 0 & 0 & 0 & 0 & \mathrm{p} & 0 & 0 & 0 & 0 & 0 & 0 & 0 & 0 & 0 & 0 & 0 & \mathrm{p} & 0 & 0 & \mathrm{p} & 0 & 0\end{array}$ $\begin{array}{llllllllllllllllllllllllllllllllllllllllll}0 & 0 & 0 & 0 & 0 & 0 & 0 & 0 & 0 & 0 & \mathrm{p} & 0 & 0 & 0 & 0 & 0 & 0 & 0 & 0 & 0 & 0 & 0 & 0 & 0 & 0 & 0 & 0 & 0 & 0\end{array}$ $\begin{array}{lllllllllllllllllllllllllllllllllllllll}0 & 0 & 0 & 0 & 0 & 0 & 0 & 0 & 0 & 0 & 0 & 0 & 0 & 0 & \mathrm{p} & \mathrm{p} & \mathrm{p} & \mathrm{p} & \mathrm{p} & \mathrm{p} & \mathrm{p} & 0 & 0 & 0 & 0 & 0 & 0 & 0 & 0\end{array}$ $\begin{array}{lllllllllllllllllllllllllllllllllllll}0 & 0 & 0 & 0 & 0 & 0 & 0 & 0 & 0 & 0 & 0 & 0 & 0 & 0 & 0 & 0 & 0 & 0 & 0 & 0 & 0 & 0 & 0 & 0 & 0 & 0 & \mathrm{p} & 0 & 0\end{array}$ $\begin{array}{llllllllllllllllllllllllllllllllllll}0 & 0 & 0 & 0 & 0 & 0 & 0 & 0 & 0 & 0 & 0 & 0 & 0 & 0 & 0 & 0 & 0 & \mathrm{p} & 0 & \mathrm{p} & 0 & 0 & 0 & \mathrm{p} & 0 & 0 & 0 & 0 & 0\end{array}$ $\begin{array}{lllllllllllllllllllllllllllllllllllll}0 & 0 & 0 & 0 & 0 & 0 & 0 & 0 & 0 & 0 & 0 & 0 & 0 & 0 & 0 & 0 & 0 & 0 & 0 & 0 & 0 & 0 & 0 & \mathrm{p} & 0 & 0 & \mathrm{p} & 0 & 0\end{array}$ $\begin{array}{lllllllllllllllllllllllllllllllllllllllll}0 & 0 & 0 & 0 & 0 & 0 & 0 & 0 & 0 & 0 & 0 & 0 & 0 & 0 & 0 & 0 & 0 & 0 & 0 & 0 & 0 & 0 & 0 & \mathrm{p} & 0 & 0 & \mathrm{p} & 0 & 0 \\ 0 & 0 & 0 & 0 & 0 & 0 & 0 & 0 & 0 & 0 & 0 & 0 & 0 & 0 & 0 & 0 & 0 & 0 & 0 & 0 & 0 & 0 & 0 & \mathrm{p} & 0 & 0 & 0 & 0 & 0\end{array}$ $\begin{array}{lllllllllllllllllllllllllllllll}0 & 0 & 0 & 0 & 0 & 0 & 0 & 0 & 0 & 0 & 0 & 0 & 0 & 0 & 0 & 0 & 0 & 0 & 0 & 0 & 0 & 0 & 0 & \mathrm{p} & 0 & 0 & 0 & 0 & 0\end{array}$ $\begin{array}{lllllllllllllllllllllllllllllll}0 & 0 & 0 & 0 & 0 & 0 & 0 & 0 & 0 & 0 & \mathrm{p} & 0 & 0 & 0 & 0 & 0 & 0 & 0 & 0 & 0 & 0 & 0 & 0 & 0 & 0 & 0 & 0 & 0 & 0\end{array}$ $\begin{array}{lllllllllllllllllllllllllllll}0 & 0 & 0 & 0 & 0 & 0 & 0 & 0 & 0 & 0 & \mathrm{p} & 0 & 0 & 0 & 0 & 0 & 0 & 0 & 0 & 0 & 0 & 0 & 0 & 0 & 0 & 0 & 0 & 0 & 0\end{array}$ $\begin{array}{lllllllllllllllllllllllllllllllll}0 & 0 & 0 & 0 & 0 & 0 & 0 & 0 & 0 & 0 & 0 & 0 & 0 & 0 & 35 & 0 & 1 & 0 & 0 & 3 & 0 & 0 & 0 & 0 & 0 & 0 & 0 & 0 & 0\end{array}$ $\begin{array}{lllllllllllllllllllllllllllllllllllllllllll}0 & 0 & 0 & 0 & 0 & 0 & 0 & 0 & 0 & 0 & 1 & 0 & 0 & 0 & 31 & 0 & 5 & 0 & 142 & 0 & 0 & 0 & 0 & 0 & 0 & 0 & 0 & 0 & 0\end{array}$ $\begin{array}{rrrrcccccccccccccccccccccccccc}0 & 0 & 0 & 0 & 50 & 0 & 0 & 1 & 0 & 0 & 0 & 0 & 0 & 0 & 0 & 0 & 0 & 0 & 0 & 2 & 12 & 29 & 10 & 0 & 0 & 6 & 0 & 0 & 22 \\ 0 & 0 & 0 & 0 & 0 & 0 & 0 & 0 & 0 & 0 & 0 & 0 & 0 & 0 & 0 & 0 & 2 & 0 & 0 & 0 & 0 & 0 & 0 & 0 & 0 & 0 & 0 & 0 & 0\end{array}$ $\begin{array}{lllllllllllllllllllllllllllllll}30 & 0 & 0 & 0 & 0 & 0 & 0 & 0 & 0 & 0 & 0 & 0 & 0 & 0 & 0 & 0 & 0 & 0 & 0 & 1 & 0 & 0 & 0 & 0 & 0 & 0 & 0 & 0 & 0\end{array}$ $\begin{array}{llllllllllllllllllllllllllllll}0 & 0 & 0 & 0 & 0 & 0 & 0 & 0 & 0 & 0 & 1 & 0 & 0 & 0 & 0 & 0 & 0 & 0 & 0 & 1 & 0 & 0 & 0 & 0 & 0 & 0 & 0 & 0 & 0\end{array}$ $\begin{array}{llllllllllllllllllllllllllllll}0 & 0 & 0 & 0 & 0 & 0 & 0 & 0 & 0 & 0 & 6 & 0 & 0 & 0 & 0 & 0 & 0 & 0 & 0 & 0 & 0 & 0 & 0 & 0 & 0 & 0 & 0 & 0 & 0\end{array}$ $\begin{array}{llllllllllllllllllllllllllllllllllllll}0 & 0 & 0 & 0 & 0 & 0 & 0 & 0 & 0 & 0 & 0 & 2 & 0 & 0 & 0 & 0 & 0 & 0 & 0 & 0 & 0 & 0 & 0 & 0 & 1 & 0 & 0 & 0 & 0\end{array}$ $\begin{array}{llllllllllllllllllllllllllllllllllllll}3 & 0 & 0 & 0 & 0 & 0 & 0 & 0 & 0 & 0 & 0 & 0 & 0 & 0 & 0 & 0 & 0 & 0 & 0 & 0 & 0 & 0 & 0 & 0 & 0 & 0 & 0 & 0 & 0\end{array}$ $\begin{array}{llllllllllllllllllllllllllllllllllllll}0 & 0 & 0 & 0 & 0 & 0 & 0 & 0 & 0 & 0 & 16 & 0 & 0 & 0 & 2 & 8 & 0 & 0 & 0 & 0 & 0 & 0 & 0 & 0 & 0 & 0 & 0 & 0 & 0\end{array}$ $\begin{array}{llllllllllllllllllllllllllllll}0 & 0 & 0 & 0 & 0 & 0 & 0 & 0 & 0 & 0 & 1 & 0 & 0 & 0 & 0 & 0 & 0 & 0 & 0 & 0 & 0 & 0 & 0 & 2 & 4 & 0 & 0 & 0 & 0\end{array}$ $\begin{array}{llllllllllllllllllllllllllllllllllllll}0 & 0 & 0 & 0 & 0 & 0 & 0 & 0 & 0 & 0 & 0 & 0 & 0 & 0 & 0 & 0 & 0 & 0 & 0 & 0 & 0 & 0 & 0 & 1 & 0 & 0 & 0 & 0 & 0\end{array}$ $\begin{array}{llllllllllllllllllllllllllllll}0 & 0 & 0 & 0 & 0 & 0 & 0 & 0 & 0 & 0 & 0 & 0 & 0 & 0 & 0 & 0 & 0 & 0 & 0 & 0 & 0 & 0 & 0 & 0 & 0 & 0 & 0 & 4 & 1\end{array}$

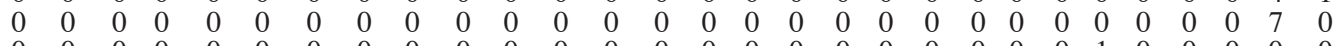
$\begin{array}{lllllllllllllllllllllllllllllllllllllll}0 & 0 & 0 & 0 & 0 & 0 & 0 & 0 & 0 & 0 & 0 & 0 & 0 & 0 & 0 & 0 & 0 & 0 & 0 & 0 & 0 & 0 & 0 & 1 & 0 & 0 & 0 & 0 & 0\end{array}$ $\begin{array}{lllllllllllllllllllllllllllllllllllll}0 & 0 & 0 & 0 & 0 & 0 & 0 & 0 & 0 & 0 & 0 & 0 & 0 & 0 & 0 & 0 & 0 & 0 & 0 & 0 & 0 & 0 & 0 & 0 & 4 & 0 & 0 & 0 & 0\end{array}$ $\begin{array}{llllllllllllllllllllllllllllllll}0 & 0 & 0 & 0 & 0 & 0 & 0 & 0 & 0 & 0 & 0 & 0 & 0 & 0 & 0 & 0 & 0 & 0 & 0 & 0 & 0 & 0 & 0 & 0 & 1 & 0 & 0 & 0 & 0 \\ 0 & 0 & 0 & 0 & 0 & 0 & 0 & 0 & 0 & 0 & 0 & 0 & 0 & 0 & 0 & 0 & 0 & 0 & 0 & 0 & 0 & 0 & 0 & 0 & 1 & 0 & 0 & 0 & 0\end{array}$ $\begin{array}{llllllllllllllllllllllllllllllll}0 & 0 & 0 & 0 & 0 & 0 & 0 & 0 & 0 & 0 & 0 & 0 & 0 & 0 & 0 & 0 & 0 & 0 & 0 & 0 & 0 & 0 & 2 & 5 & 0 & 0 & 0 & 3 & 0\end{array}$

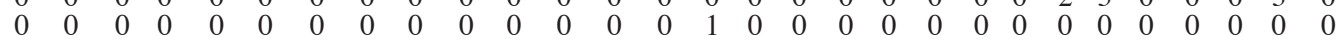
$\begin{array}{llllllllllllllllllllllllllllll}0 & 0 & 0 & 0 & 0 & 0 & 0 & 0 & 0 & 0 & 0 & 0 & 0 & 0 & 0 & 0 & 0 & 0 & 0 & 1 & 0 & 0 & 0 & 0 & 0 & 0 & 0 & 0 & 0\end{array}$ 
TABLE 2 (Cont.). - A list of marine invertebrate species found in the areas of study. Number of individuals caught with the Agassiz trawl at each station is included $(\mathrm{H}=$ South Patagonian Icefield stations; $\mathrm{E}=$ Straits of Magellan stations $)$. Colonial organisms are indicated by $\mathrm{p}=$ presence.

Species/stations number

H5 H8 H13H14 H15 H19 H22 H25 H27 H32H33sH35s H39 H40 E1 E2 E3 E4 E5 E6 E55E56 E8 E9 E10 E12 E14 E15E16

Asteroidea
Ganeria falklandica
Bathybiaster loripes
Poraniopsis echinaster
Cycethra verrucosa
Austrocidaris lorioli
Brisaster moseleyi
Echinoidea
Tripylaster philippii
Arbacia dufresnei
Pseudechinus magellanicus
Ophiuroidea
Ophiura INDET
Ophiacantha cf. pentactis
Ophiactis sp.
Ophiocten amitinum
Ophiacantha vivipara
Ophiuroglypha lymani
Gorgonocephalus chilensis
Ophiomyxa vivipara
Ophiactis asperula
Astrotoma agassizii
Homalophiura inornata
Ophioscolex nutrix
Homalophiura sp.
Holothuroidea
Hemoiedema spectabilis
Psolus patagonicus
Pseudocnus leoninus
dubiosus
Holothuroidea sp 1
Holothuroidea sp 2
Holothuroidea sp 3

$\begin{array}{cccccccccccccccccccccccccccccc}0 & 0 & 0 & 0 & 0 & 0 & 0 & 0 & 0 & 0 & 0 & 0 & 0 & 0 & 0 & 4 & 0 & 0 & 1 & 0 & 0 & 0 & 0 & 0 & 0 & 0 & 0 & 0 & 0 \\ 0 & 0 & 0 & 0 & 0 & 0 & 0 & 0 & 0 & 0 & 0 & 0 & 0 & 0 & 0 & 0 & 0 & 0 & 0 & 0 & 3 & 0 & 2 & 0 & 0 & 1 & 0 & 0 & 4 \\ 0 & 0 & 0 & 0 & 0 & 0 & 0 & 0 & 0 & 0 & 2 & 0 & 0 & 0 & 0 & 0 & 0 & 0 & 0 & 0 & 0 & 0 & 0 & 0 & 0 & 0 & 0 & 0 & 0 \\ 0 & 0 & 0 & 0 & 18 & 0 & 0 & 0 & 0 & 0 & 0 & 0 & 0 & 0 & 2 & 0 & 1 & 10 & 0 & 0 & 0 & 0 & 0 & 0 & 4 & 0 & 0 & 0 & 0 \\ 0 & 0 & 0 & 0 & 0 & 0 & 0 & 0 & 0 & 0 & 0 & 0 & 0 & 0 & 2 & 0 & 0 & 0 & 0 & 0 & 0 & 0 & 0 & 2 & 0 & 0 & 0 & 0 & 0 \\ 0 & 0 & 0 & 0 & 0 & 0 & 0 & 0 & 0 & 0 & 0 & 0 & 0 & 0 & 0 & 0 & 0 & 0 & 0 & 0 & 1 & 0 & 0 & 0 & 0 & 0 & 0 & 0 & 0\end{array}$

$\begin{array}{lllllllllllllllllllllllllllllll}0 & 0 & 0 & 0 & 0 & 0 & 0 & 0 & 0 & 0 & 0 & 0 & 0 & 0 & 0 & 0 & 0 & 0 & 0 & 0 & 0 & 14 & 0 & 0 & 0 & 0 & 0 & 0 & 2\end{array}$ $\begin{array}{lllllllllllllllllllllllllllllllllllllllll}0 & 0 & 1 & 0 & 0 & 0 & 0 & 0 & 0 & 0 & 20 & 0 & 0 & 0 & 0 & 0 & 0 & 0 & 0 & 0 & 0 & 0 & 0 & 0 & 1 & 0 & 0 & 0 & 0\end{array}$ $\begin{array}{lllllllllllllllllllllllllllllllllllll}0 & 0 & 0 & 0 & 0 & 0 & 2 & 0 & 0 & 0 & 8 & 4 & 24 & 0 & 0 & 6 & 1 & 0 & 11 & 20 & 0 & 0 & 0 & 5 & 9 & 0 & 0 & 0 & 0\end{array}$ Ophiuroidea

$\begin{array}{lllllllllllllllllllllllllllllllllllllll}0 & 0 & 0 & 0 & 0 & 0 & 0 & 0 & 0 & 0 & 0 & 0 & 0 & 0 & 0 & 0 & 0 & 0 & 1 & 1 & 0 & 0 & 0 & 0 & 0 & 0 & 0 & 0 & 0\end{array}$ $\begin{array}{lllllllllllllllllllllllllllllllllll}\text { Ophiactis sp. } & 0 & 0 & 0 & 0 & 0 & 0 & 0 & 0 & 0 & 0 & 111 & 0 & 0 & 0 & 0 & 0 & 0 & 0 & 0 & 0 & 0 & 0 & 0 & 0 & 0 & 0 & 0 & 0 & 0\end{array}$ $\begin{array}{llllllllllllllllllllllllllllllllll}\text { Ophiocten amitinum } & 0 & 0 & 0 & 0 & 0 & 0 & 0 & 0 & 0 & 0 & 0 & 13 & 0 & 0 & 0 & 0 & 0 & 0 & 0 & 0 & 0 & 0 & 0 & 0 & 0 & 0 & 0 & 0 & 0\end{array}$

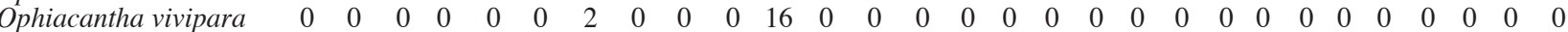

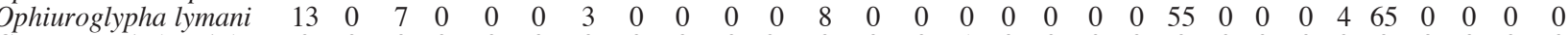
$\begin{array}{lllllllllllllllllllllllllllllllllllllll}0 & 0 & 0 & 0 & 0 & 0 & 0 & 3 & 0 & 0 & 0 & 27 & 0 & 0 & 0 & 1 & 0 & 0 & 0 & 0 & 0 & 0 & 0 & 0 & 0 & 0 & 0 & 0 & 0 & 0\end{array}$ $\begin{array}{lllllllllllllllllllllllllllllllll}\text { Ophiactis asperula } & 0 & 0 & 0 & 0 & 0 & 0 & 0 & 0 & 0 & 0 & 0 & 0 & 0 & 0 & 0 & 0 & 5 & 5 & 0 & 0 & 0 & 0 & 0 & 0 & 0 & 0 & 0 & 1 & 1\end{array}$ $\begin{array}{llllllllllllllllllllllllllllllllllll}\text { Astrotoma agassizii } & 0 & 0 & 0 & 0 & 0 & 0 & 0 & 3 & 0 & 0 & 0 & 0 & 0 & 0 & 0 & 0 & 0 & 0 & 0 & 0 & 0 & 0 & 0 & 0 & 0 & 0 & 0 & 0 & 0\end{array}$

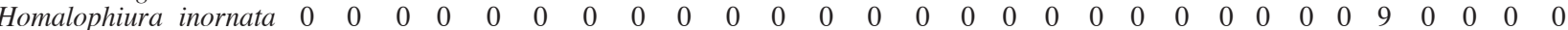
$\begin{array}{lllllllllllllllllllllllllllllllllll}\text { Ophioscolex nutrix } & 0 & 0 & 0 & 0 & 0 & 0 & 2 & 0 & 0 & 0 & 0 & 0 & 0 & 0 & 0 & 0 & 0 & 0 & 0 & 0 & 0 & 0 & 0 & 0 & 0 & 0 & 0 & 0 & 0\end{array}$ Homalophiura sp. $\begin{array}{lllllllllllllllllllllllllllllllllllllllllll}0 & 0 & 0 & 0 & 0 & 0 & 0 & 0 & 0 & 0 & 0 & 0 & 0 & 0 & 0 & 0 & 0 & 0 & 0 & 0 & 0 & 0 & 0 & 0 & 0 & 0 & 0 & 0 & 0\end{array}$ $\begin{array}{llllllllllllllllllllllllllllllllllll}0 & 0 & 0 & 0 & 0 & 0 & 0 & 0 & 0 & 0 & 0 & 0 & 0 & 0 & 0 & 1 & 0 & 0 & 0 & 0 & 0 & 0 & 0 & 0 & 0 & 0 & 0 & 0 & 0\end{array}$

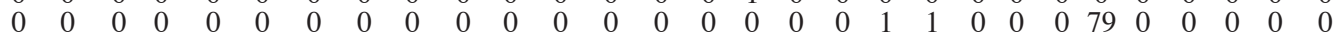
$\begin{array}{llllllllllllllllllllllllllllll}0 & 0 & 0 & 0 & 0 & 0 & 0 & 0 & 0 & 0 & 0 & 0 & 0 & 0 & 0 & 0 & 1 & 0 & 0 & 0 & 0 & 0 & 0 & 0 & 0 & 0 & 0 & 0 & 0\end{array}$

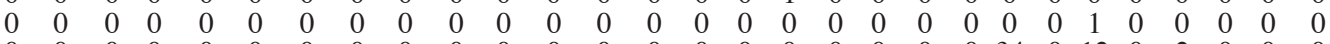

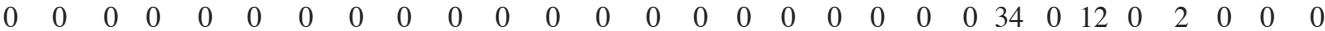
Holothuroidea sp 3

with the biggest catches were the two stations in Paso Ancho (stations C5 and C6; see Fig. 1), Isla Wood (C9), and Paso Tortuoso (C10). The most frequently occurring group of specimens in the entire SM area was Brachiopoda, with the articulate brachiopod Magellania venosa as the most prominent species. Another dominant group was the Asteroidea, mainly Ctenodiscus procurator and Cycethra verrucosa. The ophiuroids Ophiuroglypha lymani and Ophiactis asperula, and the holothurian Psolus patagonicus were also relatively abundant at some sampling stations.

Differences between the subgroups determined on the basis of samples from the Atlantic (eastern) and Pacific (western) entrance of the Strait of Magellan appear to be defined mainly by the occurrence of echinoderms and brachiopods. In the western area, the asteroids Ctenodiscus procurator and Bathybiaster loripes and the isopod Acanthoserolis schythei dominated the samples, whereas at the eastern entrance Magellania venosa and Terebratella dorsata (brachiopods), Ophiactis asperula (ophiuroid) and Cycethra verrucosa (asteroid) were the dominant species.
A similar analysis with the SPI sampling sites showed no clear-cut relation to geographical positions (Fig 2c). Agassiz trawl catches were larger at sampling sites situated in channels (e.g. Canal Oeste and Paso del Indio) and also at the entrance of the Penguin Fjord. The remaining stations, mainly those in the fjords, showed smaller catches as compared with the relatively big ones obtained at sites less influenced by glaciers. The bivalve Camptonectus (P.) subhyalinus was the most abundant species off the SPI, followed by the ophiuroids Ophiactis sp., Ophiuroglypha lymani and Gorgonocephalus chilensis. Other dominant species in this area were the polychaetes Polyeunoa laevis and Harmothoe campoglacialis, and the asteroids Ctenodiscus procurator and Luidiaster planatus.

According to the results from the BIOENV analysis, no relationship was found between the benthic distribution patterns observed and the abiotic parameters in the SPI area. A similar result was obtained for the SM area; however, a low level of correlation was obtained between the faunal composition, the hydrodynamic regime and suspended particulate matter $(\mathrm{R}=0.040 ; \mathrm{p}=0.24)$. 

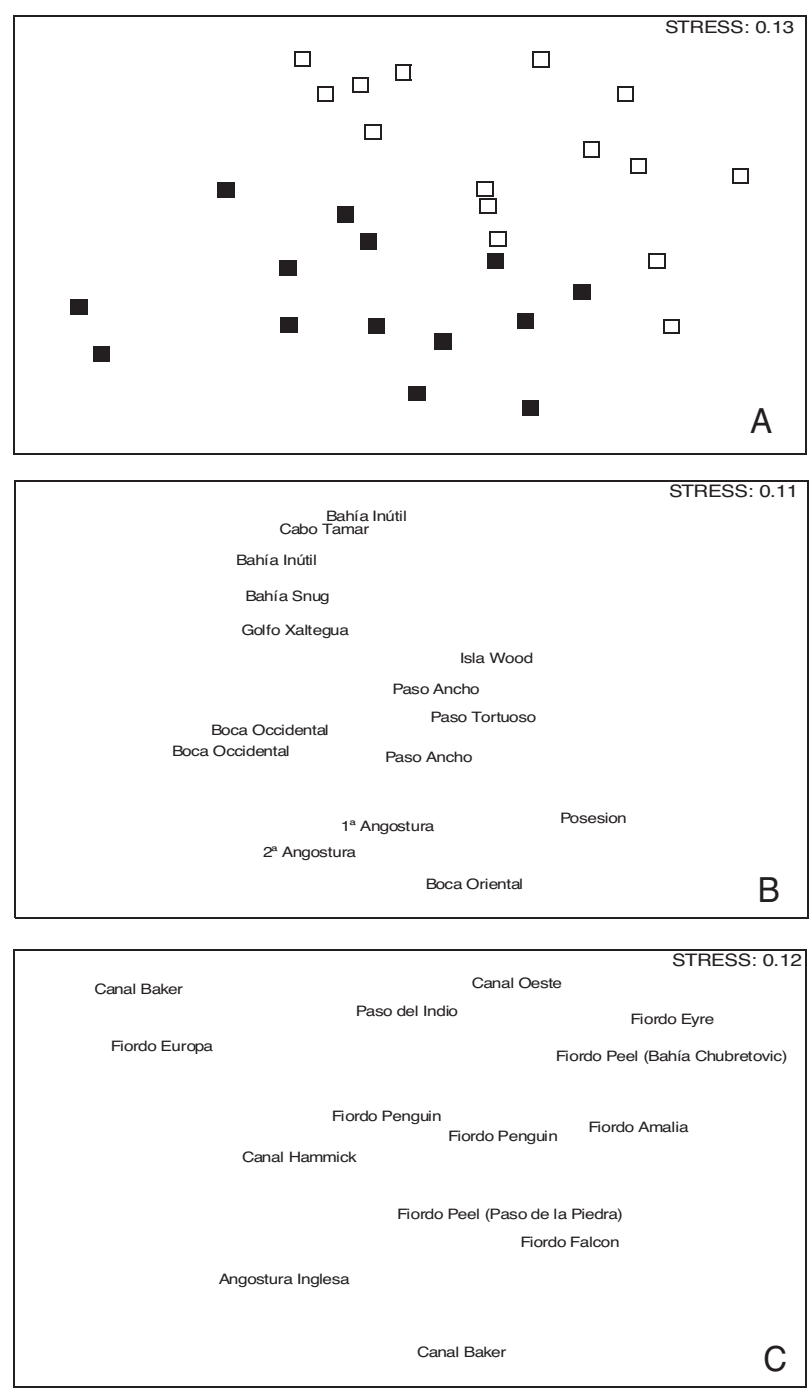

FIG. 2. - MDS plots of macrobenthic assemblages in the Magellan region (A; $\square=$ SPI stations, $\square=$ EM stations), the Straits of Magellan (B) and the South Patagonian Icefield (C), based on the Bray-Curtis similarity index with presence/absence data.

\section{DISCUSSION}

This study is the first attempt to describe and compare macrobenthic associations on a species level in the south Chilean glacial fjords and channels. However, due to the sampling gear used (Agassiz trawl), some caution is needed for the interpretation of the resulting ecological patterns. The study of macrozoobenthic communities by trawls can be considered to give only a first insight into the distribution of epibenthic communities, as mentioned by Arnaud et al. (1998), but it represents a fairly good picture of the variety of benthic species living in an ecosystem. Further quantitative research on species composition, biotic and abiotic variables and their temporal and spatial variability is necessary for a more global understanding of community patterns and processes occurring in these fjords and channels.

A low number of specimens was an outstanding feature at all the sampling stations, especially at those stations located in the SPI area. Distribution patterns in the SM area were found to be more homogenous, as is obvious also from extensive quantitative analyses on a major taxon level. The lowest average abundance $\left(625 \mathrm{ind} / \mathrm{m}^{2}\right)$ was observed at sampling stations in the South Patagonian Icefield, increasing significantly southwards to a maximum $\left(3,972 \mathrm{ind} / \mathrm{m}^{2}\right)$ on the continental shelf off the eastern entrance of the Beagle Channel. The Straits of Magellan showed intermediate values $\left(1,591 \mathrm{ind} / \mathrm{m}^{2}\right)$. These differences are attributed to certain environmental properties of the areas, mainly those related to the stability of the bottom sediments. Silva and Prego (2002) indicated that glaciers provide cold oligotrophic waters rich in silt to the inlets, features which may explain the scarcity of benthic abundance and biomass production, as was suggested for the phytoplankton scarcity found in several of the fjords considered in our study (Vera et al., 1996).

However, lack of repeated study in this ecosystem, including seasonal and long-term fluctuations of abundance and environmental abiotic parameters, make a more comprehensive analysis and explanation of the observed trends rather difficult, especially for the SPI. For instance, melting of glaciers during the austral spring (September-early December) and summer (December-March) might cause a strong sedimentary input of inorganic matter to the bottom, producing additional disturbance in the benthic regime, and consequently a decrease in animal abundance. Sedimentation has been suggested as one of the important factors determining epifaunal distribution at Antarctic coastal glaciers (e.g. Dawber and Powell, 1997), and more specifically in Potter Cove (Kind George Island), where benthic colonisation (ascidians instead of sponges) is strongly influenced by inorganic siltation from the glacier (Klöser et al., 1994; Kowalke and Abele 1998; Sahade et al. 1998; Tatián et al. 1998). Similar results have also been obtained in the Canadian Arctic and Alaskan fjords, where epifauna is restricted to areas with low sedimentation rates (Evans et al., 1980; Carney et al., 1999).

An important additional difference to sites located in the SPI area is the higher amount of inorganic carbon in surface sediments of the Straits of Magel- 
TABLE 3. - Average dissimilarity (AvDiss) and ratio between AvDiss and its corresponding standard deviation (Diss/SD) between SPI (South Patagonian Icefield) and SM (Straits of Magellan) groups. Species are ranked in decreasing contribution (\%) to dissimilarity values. Cum=cumulative percentage. Average dissimilarity $=97.35$

\begin{tabular}{|c|c|c|c|c|c|c|}
\hline Species & $\begin{array}{l}\text { Group SPI } \\
\text { AvAbund }\end{array}$ & $\begin{array}{c}\text { Group SSM } \\
\text { AvAbund }\end{array}$ & AvDiss & Diss/SD & Contrib\% & Cum \\
\hline Ctenodiscus procurator & 3.64 & 5.40 & 10.45 & 0.65 & 10.71 & 10.71 \\
\hline Ophiuroglypha lymani & 2.21 & 8.27 & 7.33 & 0.57 & 7.52 & 18.23 \\
\hline Magellania venosa & 0.07 & 11.87 & 6.51 & 0.39 & 6.68 & 24.91 \\
\hline Pseudechinus magellanicus & 2.71 & 3.47 & 5.02 & 0.52 & 5.15 & 30.05 \\
\hline Acanthoserolis schythei & 0.00 & 3.93 & 4.94 & 0.36 & 5.07 & 35.12 \\
\hline Camptonectus(Pallioleum)subhyalinus & 13.64 & 0.00 & 4.54 & 0.27 & 4.65 & 39.77 \\
\hline Dentalium majorinum & 0.43 & 1.53 & 3.40 & 0.42 & 3.49 & 43.26 \\
\hline Limopsis marionensis & 0.21 & 5.13 & 3.19 & 0.36 & 3.27 & 46.53 \\
\hline Psolus patagonicus & 0.00 & 5.40 & 3.16 & 0.26 & 3.24 & 49.77 \\
\hline Holothuroidea sp 2 & 0.00 & 3.20 & 3.05 & 0.36 & 3.13 & 52.90 \\
\hline Cycethra verrucosa & 1.29 & 1.13 & 2.91 & 0.36 & 2.99 & 55.88 \\
\hline Ophiactis sp. & 7.93 & 0.00 & 2.36 & 0.27 & 2.42 & 58.30 \\
\hline Terebratella dorsata & 0.00 & 2.60 & 2.35 & 0.28 & 2.41 & 60.71 \\
\hline Cheiraster (Luidiaster) planeta & 2.14 & 0.07 & 2.31 & 0.26 & 2.37 & 63.07 \\
\hline Ophiactis asperula & 0.00 & 0.80 & 1.73 & 0.37 & 1.77 & 64.85 \\
\hline Cosmasterias lurida & 1.14 & 0.67 & 1.65 & 0.31 & 1.70 & 66.54 \\
\hline Leanira quatrefagesi & 1.36 & 0.00 & 1.61 & 0.59 & 1.65 & 68.19 \\
\hline Bathybiaster loripes & 0.00 & 0.67 & 1.36 & 0.49 & 1.40 & 69.59 \\
\hline Ennucula grayi & 0.64 & 0.40 & 1.29 & 0.28 & 1.32 & 70.91 \\
\hline Eurypodius latreillei & 0.64 & 0.73 & 1.19 & 0.42 & 1.22 & 72.13 \\
\hline Trypilaster philippi & 0.00 & 1.07 & 1.10 & 0.31 & 1.13 & 73.26 \\
\hline Ophiocten namitinum & 0.93 & 0.00 & 1.08 & 0.25 & 1.11 & 74.37 \\
\hline Gorgonocephalus chilensis & 2.14 & 0.07 & 1.04 & 0.39 & 1.06 & 75.43 \\
\hline Polyeunoa laevis & 2.57 & 0.00 & 1.03 & 0.35 & 1.06 & 76.49 \\
\hline Pista cristata & 0.57 & 0.00 & 0.86 & 0.25 & 0.88 & 77.37 \\
\hline Maldane sarsi & 0.43 & 0.00 & 0.85 & 0.42 & 0.88 & 78.25 \\
\hline Libidoclaea smithi & 0.36 & 0.13 & 0.79 & 0.29 & 0.81 & 79.06 \\
\hline Crepidula dilatata & 0.00 & 0.40 & 0.76 & 0.24 & 0.78 & 79.84 \\
\hline Ophiacantha $c f$. pentactis & 0.64 & 0.00 & 0.75 & 0.25 & 0.77 & 80.61 \\
\hline Cyclocardia velutinus & 0.14 & 1.20 & 0.75 & 0.35 & 0.77 & 81.37 \\
\hline Abyssoninoe abbyssorum & 0.43 & 0.00 & 0.73 & 0.31 & 0.75 & 82.12 \\
\hline Sternaspis scutata & 1.00 & 0.00 & 0.71 & 0.33 & 0.73 & 82.85 \\
\hline Porania antarctica magellanica & 0.00 & 10.67 & 10.66 & 0.45 & 0.67 & 83.53 \\
\hline Laneria falklandica & 0.00 & 0.33 & 0.64 & 0.24 & 0.66 & 84.18 \\
\hline Ophiacantha vivipara & 1.29 & 0.00 & 0.62 & 0.37 & 0.63 & 84.81 \\
\hline Berthella platei & 0.07 & 0.53 & 0.61 & 0.31 & 0.63 & 85.44 \\
\hline Aulacomya ater & 0.00 & 0.40 & 0.60 & 0.34 & 0.61 & 86.06 \\
\hline Arbacia dufresnei & 1.50 & 0.07 & 0.58 & 0.36 & 0.60 & 86.65 \\
\hline Munida subrugosa & 0.21 & 0.13 & 0.55 & 0.41 & 0.57 & 87.22 \\
\hline Campylonotus semistriatus & 0.00 & 0.87 & 0.52 & 0.34 & 0.54 & 87.75 \\
\hline Calyptraster tenuissimus & 0.00 & 0.47 & 0.51 & 0.25 & 0.52 & 88.28 \\
\hline Halicarcinus planatus & 0.00 & 0.27 & 0.51 & 0.24 & 0.52 & 88.80 \\
\hline Harmothoe campoglacialis & 1.64 & 0.00 & 0.49 & 0.27 & 0.50 & 89.30 \\
\hline Chlamys patagonicus & 1.21 & 0.27 & 0.46 & 0.34 & 0.48 & 89.77 \\
\hline Homalophiura inornata & 0.00 & 0.60 & 0.41 & 0.25 & 0.42 & 90.20 \\
\hline
\end{tabular}

lan (Silva and Prego, 2002), which according to Brambati et al. (1991) can be attributed to the presence of calcite in the remains of bivalves, gastropods, crustaceans and foraminiferans. Unfortunately, information on chemical and physical processes of pelagobenthic coupling (Cattaneo-Vietti et al., 1999) and in the bottom sediments is scarce for the area of study, and this represents a limiting factor to explain the (dis)similarities we found between the study areas. More focused research on inter-annual variability of biotic parameters and their relationship with variability in sediments features are clearly needed for the Magellan region. Recently, Ríos et al. (2003) reported for the Straits of Magellan a significant seasonal abundance pat- tern, with variations in species richness and diversity in areas at the eastern SM entrance, and with maximum values observed in the austral summer period. Presently, this area is not affected by the direct influence of glacial processes. All these features suggest very intricate ecological dynamics in the benthic communities associated with the Chilean channel and fjord complex.

The most interesting result was the clear difference in species composition found between the South Patagonian Icefield and the Straits of Magellan. Recently, both areas have been separated into two different sectors according to carbon and nitrogen concentrations and distributions in the superficial sediment layers of the bottom (Silva and Prego, 
2002). In addition, an east (glacially affected zone) and west (oceanically influenced zone) segregation in the SPI was identified. However, our biotic data do not indicate any clear relationship with the abiotic parameters determined by Silva and Prego (2002): the differences could be primarily explained by the occurrence of markedly different habitat conditions and gradients caused by the glaciers.

The discriminant invertebrate species determined for the SPI area are typical of the prevailing substrate (mud in the fjords and sand in the channels), but also for the presumably considerable disturbance produced by high sedimentation towards the bottom. These facts may explain the larger catches both in channels (e.g. Canal Oeste) and at the entrance of the fjords (e.g. Seno Pinguiino). One of the discriminant genera in the SPI area, the ophiuroid Ophiactis sp. was collected at Canal Oeste sampling station, and has been frequently mentioned as being common in communities characterised by oligospecificity with dominance of suspension feeders, in which hydrodynamics represent both the most important limiting factor and the main carrier of organic matter (Giacobbe and Rinelli, 1991). Other species characterising this area were the carnivorous polynoids Polyeunoa laevis (dominant at the entrance of Seno Pinguiino) and Harmothoe campoglacialis (Canal Oeste), and the ophiourid Gorgonocephalus chilensis (Canal Oeste). P. laevis has a circumpolar distribution, and is a dominant species in assemblages defined for deep water and hard bottoms off Subantarctic islands (Guillet, 1989). $H$. campoglacialis is a new, recently-identified species (Hilbig and Montiel, 2000) with a wide distribution range in the southern part of Chile, inhabiting mud and fine sand of fjords, channels and even microhabitats such as holdfasts of the kelp Macrocystis pyrifera (Montiel et al., 2004). Species of the genus Gorgonocephalus are considered as "fortuitous predatory suspension feeders" adapted to life in strong currents (Emson et al., 1991).

The SM represent a more diverse and probably less disturbed habitat for diverse benthic communities with higher organism densities. Furthermore, our data suggest the occurrence of separate subgroups of stations along the Straits with differences in community composition, e.g. at both entrances of SM. Such variability did not become evident in the comparison of mega-epibenthos distribution across the Straits by Gutt et al. (1999). In the SM area the most significant species regarding the discrimination from the SPI area (Ctenodiscus procurator, Ophiuroglypha lymani and Magellania venosa) have been previously reported as characteristic species of benthic communities in the Straits of Magellan (Dahm, 1999; Larraín et al., 1999; Gutt et al., 1999). The genus Ctenodiscus is considered to be a non-selective deposit feeder (Shick et al., 1981), whereas O. lymani is an omnivorous species and feeds mainly on crustaceans, the organic content of sediment and phytodetritus (Dahm, 1999). The brachiopod genus Magellania has been described as a dominant and characteristic filter feeder on deep soft substrates in the Subantarctic Prince Edward Islands (Branch et $a l ., 1993)$ and on the continental shelf of the high Antarctic Lazarev Sea (Brey et al., 1995).

\section{ACKNOWLEDGEMENTS}

The following experts assisted in the identification of different taxa: Boris Sirenko (polyplacophora), Igor Smirnov (ophiuroids), Hugo Moyano (bryozoa), Christoph Held (isopods), Erika Mutschke (echinoderms) and Américo Montiel (polychaetes). The scientific cruises to obtain the benthic data analysed in this article (CIMAR-FIORDO III and VII) were organised and developed by the Comité Científico Nacional (CONA) on board the R/V "Vidal Gormaz". The support of the R/V "Vidal Gormaz" crew is greatly appreciated. Jorge Ramírez V. (Laboratorio de Hidrobiología, Instituto de la Patagonia, Universidad de Magallanes) was an important help during all the fieldwork and also in the laboratory, and we extend our gratitude for his effort. We thank the Universidad de Magallanes (Punta Arenas, Chile) and CONA for partial financial support.

\section{REFERENCES}

Ahumada, R., A. Rudolph and N. Silva. - 1996. Contenido de carbono total, carbono orgánico, carbono inorgánico, nitrógeno orgánico y fósforo total, en los sedimentos de los fiordos de Campo de Hielo Sur. Cienc. Tecnol. Mar., 19: 123-132.

Arnaud, P.M., C.M. López, I. Olaso, F. R. Ramil, A.A. RamosEsplá and A. Ramos. - 1998. Semi-quantitative study of macrobenthic fauna in the region of the South Shetland islands and the Antarctic Peninsula. Polar Biol., 19: 160-166.

Arntz, W. - 1999. Magellan - Antarctic: Ecosystems that drifted apart. Summary Review. In: W.E. Arntz and C. Ríos (eds.), Magellan-Antarctic. Ecosystems that Drifted Apart. Sci. Mar., 63(Suppl. 1): 503-511.

Arntz, W.E. and C. Ríos (eds.). - 1999. Magellan-Antarctic. Ecosystems that drifted Apart. Sci. Mar., 63(Suppl. 1): 1-518.

Arntz, W.E. and M. Gorny (eds.). - 1996. Cruise report of the Chilean-German-Italian Magellan "Victor Hensen” campaign in 1994. Ber. Polarforsch., 190: 1-113.

Arntz, W.E., T. Brey and V.A. Gallardo. - 1994. Antarctic zooben- 
thos. Oceanogr. Mar. Biol. Ann. Rev., 32: 241-304.

Brambati, A., G. Fontolan and U. Simeoni. - 1991. Recent sediments and sedimentological processes in the Straits of Magellan. Boll. Oceanol. Teor. Appl., 2-3: 217-260.

Branch, G.M., C.G. Attwood, D. Gianakouras and M.L. Branch. 1993. Patterns in the benthic communities on the shelf of the Sub-Antarctic Prince Edward Islands. Polar Biol., 13: 23-34.

Brattström, H. and A. Johanssen. - 1983. Ecological and regional zoogeography of the marine benthic fauna of Chile. Sarsia, 68: 289-339.

Brey, T. and D. Gerdes. - 1999. Benthic community productivity in the Magellan region and in the Weddell Sea. In: W.E. Arntz and C. Ríos (eds.), Magellan-Antarctic. Ecosystems that drifted apart. Sci. Mar., 63(Suppl. 1): 145-148.

Brey, T., L.S. Peck, J. Gutt, S. Hain and W.E. Arntz. - 1995. Population dynamics of Magellania fragilis, a brachiopod dominating a mixed-bottom macrobenthic assemblage on the Antarctic shelf. J. Mar. Biol. Assoc. U.K., 75: 857-869.

Camus, P.A. - 2001. Biogeografía marina de Chile continental Rev. Chil. Hist. Nat., 74: 587-617.

Carney, D., J.S. Oliver and C. Armstrong. - 1999. Sedimentation and composition of wall communities in Alaskan fjords. Polar Biol., 22: 38-49

Cattaneo-Vietti, R., M. Chiantore, C. Misic, P. Povero and M. Fabiano. -1999 . The role of pelagic-benthic coupling in structuring littoral benthic communities at terra Nova Bay (Ross Sea) and in the Straits of Magellan. In: W.E. Arntz and C. Ríos (eds.), Magellan-Antarctic. Ecosystems that drifted apart. Sci. Mar., 63(Suppl. 1): 113-121.

Clarke, A. - 1992. Is there a latitudinal diversity cline in the sea? Trends Ecol. Evol., 7: 286-287.

Clarke, K.R. and R.M. Warwick. - 1994. Change in marine communities: an approach to statistical analysis and interpretation. Plymouth: Plymouth Marine Laboratory.

Clarke, K.R. and R.N. Gorley. - 2001. PRIMER v5: User manual/tutorial. PRIMER-E: Plymouth.

Dahm, C. - 1999. Ophiuroids (Echinodermata) of South Chile and the Antarctic: taxonomy, biomass, diet and growth of dominant species. In: W.E. Arntz and C. Ríos (eds.), Magellan-Antarctic. Ecosystems that drifted apart. Sci. Mar., 63(Suppl. 1): 427-432.

Dawber, M. and R.D. Powell. - 1997. Epifaunal distribution at Antarctic marine-ending glaciers: influence of ice dynamics and sedimentation. In: C.A. Ricci (ed.), The Antarctic region: Geological evolution and processes. pp. 875-884. Terra Antarctica Publication, Siena, Italy.

Emson, R.H., P.V. Mladenov and K. Barrow. - 1991. The feeding mechanism of the basket star Gorgonocephalus arcticus. Can. J. Zool., 69: 449-455.

Evans, R.A., B. Gulliksen and O.K. Sandes. - 1980. The effect of sedimentation on rocky bottom organisms in Balsfjord, northern Norway. In: H.J. Freeland, D.M. Farmer and C.D. Levings (eds.), Fjord oceanography. pp 603-607. Plenum, New York.

Fontolan, G. and S. Panella. - 1991. Distribution and chemistry (Fe, $\mathrm{Zn}, \mathrm{Pb}, \mathrm{Cu}, \mathrm{Cd}$, Chlorophyll a) of suspended particulate matter in the Straits of Magellan: Austral Spring 1989. Boll. Oceanol. Teor. Appl., 2-3: 179-192.

Forcelli, D. - 2000. Moluscos magallánicos. Guía de moluscos de Patagonia y sur de Chile. Vásquez Massini, Buenos Aires.

Gallardo, V.A., O. Ferretti and H.I. Moyano (eds.). - 1992. Oceanografía en Antártica. ENEA-Centro EULA-Chile, Concepción.

Gerdes, D. and A. Montiel. - 1999. Distribution patterns of macrozoobenthos: a comparison between the Magellan region and the Weddell Sea (Antarctic). In: W.E. Arntz and C. Ríos (eds.), Magellan-Antarctic. Ecosystems that drifted apart. Sci. Mar. 63(Suppl. 1): 35-38

Giacobbe, S. and P. Rinelli. - 1991. Ecological notes on Ophiactis balli (Thomson) from populations of Errina aspera in the Straits of Messina. Echinoderms Res.: 202.

Gray, J.S. - 2001a. Antarctic marine benthic biodiversity in a world-wide latitudinal context. Polar Biol., 24: 633-641.

Gray, J.S. - 2001b. Marine diversity: the paradigms in patterns of species richness examined. Sci. Mar., 65(Suppl. 2): 41-56.

Guillet, P. - 1989. Biogeography and polychaete assemblages from Subantarctic islands (Indian Ocean): Marion Dufresne MD/08 Benthos Expedition to Marion, Prince Edward and Crozet islands. Bull. Mar. Sci., 48: 358-368.

Gulliksen, B., B. Holta and K.J. Jakola. - 1985. The soft bottom fauna in Van Mijenfjord and Raudfjord, Svalbard. In: J. Gray and M.E. Christiansen (eds.), Marine biology of polar regions and effects of stress on marine organisms. pp. 199-215. Wiley, Oslo.

Gutt, J., E. Helsen, W.E. Arntz and A. Buschmann. - 1999. Biodiversity and community structure of the megaepibenthos in the Magellan region (South America). In: W.E. Arntz and C. Ríos (eds.), Magellan-Antarctic. Ecosystems that drifted apart. Sci. Mar., 63(Suppl. 1): 155-170.

Hilbig, B. and A. Montiel. - 2000. Harmothoe campoglacialis sp. nov. (Polychaeta: Polynoidae), a new scaleworm from the Magellan Region, Chile. Mitt. Hamb. Zool. Mus. Inst., 97:5-12.

Holte, B. and B. Gulliksen. - 1998. Common macrofaunal dominant species in the sediments of some north Norwegian and Svalbard glacial fjords. Polar Biol., 19: 375-382.

Hulton, N.R.J., R.S. Purves, R.D. McCulloch, D.E. Sugden and M.J. Bentley. - 2002. The last glacial maximum and deglaciation in South South America. Quat. Sci. Rev., 21: 233-241.

Jazdzewski, K., C. de Broyer, M. Pudlarz and D. Zielinski. - 2001. Seasonal fluctuations of vagile benthos in the uppermost sublittoral of a maritime Antarctic fjord. Polar Biol., 24: 910-917.

Kendall, M.A., S. Widdicombe and J.M. Weslawski. - 2003. A multiscale study of the biodiversity of the benthic infauna of the highlatitude Kongsfjord, Svalbard. Polar Biol., 26: 383-388.

Klöser, H., G. Mercuri, F. Laturnus, M.L. Quartino and C. Wiencke. - 1994. On the competitive balance of macroalgae at Potter Cove (King George Island, south Shetland. Polar Biol. 14: 11-16.

Kowalke, J. and D. Abele. - 1998. A first record of the soft bottom infauna community of Potter Cove. Ber. Polarforsch., 299: 106-112.

Kowalke, J., M. Tatián, R. Sahade and W.E. Arntz. - 2001. Production and respiration of Antarctic ascidians. Polar Biol., 24: 663-669.

Lancellotti, D.A. and J.A. Vásquez. - 2000. Zoogeografía de macroinvertebrados de la costa de Chile: contribución para la conservación marina. Rev. Chil. Hist. Nat.,73: 99-129.

Larsen, L-H. - 1997. Soft-bottom macroinvertebrate fauna of north Norwegian coastal waters with particular reference to sillbasins. Part one: Bottom topography and species diversity. Hydrobiology, 355: 101-113.

Larraín, A., E. Mutschke, A. Riveros and E. Solar. - 1999. Preliminary report on Echinoidea and Asteroidea (Echinodermata) of the joint Chilean-German-Italian Magellan "Victor Hensen" campaign, 17 October-25 November 1994. In: W.E. Arntz and C. Ríos (eds.), Magellan-Antarctic. Ecosystems that drifted apart. Sci. Mar., 63(Suppl. 1): 433-438.

Montiel, A., D. Gerdes and C. Ríos. - 2001. Distribución y abundancia del macrozoobentos en una microcuenca marina submareal del Estrecho de Magallanes, Chile. Ans. Inst. Pat., Serie Cs. Nat., 29: 117-133.

Montiel, A., C. Ríos, E. Mutschke and N. Rozbaczylo. - 2004. Poliquetos de fiordos y canales adyacentes al Campo de Hielo Patagónico Sur, Chile (Annelida, Polychaeta). Cienc. Tecnol. Mar., 27: 49-67.

Moreno, P.I., T.V. Lowell, G.L. Jacobson Jr. and G.H. Denton. 1999. Abrupt vegetation and climate changes during the last glacial maximum and the last termination in the Chilean Lake District: a case study from Canal de la Puntilla $\left(41^{\circ} \mathrm{S}\right)$ Geografiska Annaler, 81A: 285-311.

Pearson, T.H. - 1980. Macrobenthos in fjords. In: H.J. Freeland, D.M. Farmer and C.E. Leving (eds.), Fjord oceanography. pp 569-602. Plenum Press, New York.

Piepenburg, D., M.K. Schmid and D. Gerdes. - 2002. The benthos off King George Island (South Shetland Islands, Antarctica): Further evidence for a lack of a latitudinal biomass cline in the Southern Ocean. Polar Biol., 25: 146-158.

Retamal, M.A. - 1974. Contribución al conocimiento de los crustáceos decápodos de la región Magallánica. Gayana (Zool.), 31: 1-23.

Ríos, C., E. Mutschke and E. Morrison. - 2003. Biodiversidad bentónica sublitoral en el Estrecho de Magallanes. Rev. Biol. Mar. Oceanog., 38: 1-12.

Sahade, R., M. Tatián, J. Kowalke, S. Kühne and G.B. Esnal. 1998. Epifaunal communities in Potter Cove, King George Island, Antarctica. Ber. Polarforsch., 299: 123-131.

Shick, J.M., K.C. Edwards and J.H. Dearborn. - 1981. Physiological ecology of the deposit-feeding sea star Ctenodiscus crispatus: ciliated surfaces and animal-sediment interactions. Mar. 
Ecol. Progr. Ser., 5: 165-185.

Silva, N. and R. Prego. - 2002. Carbon and nitrogen spatial segregation and stoichiometry in the surface sediments of South Chilean inlets $\left(41^{\circ}-56^{\circ} \mathrm{S}\right)$. Estuar. Coast. Shelf Sci., 55: 763-775.

Tatián, M., R. Sahade, M.E. Doncet and G.B. Esnal. - 1998. Some aspects of Antarctic ascidians (Tunicata, Ascidacea) of Potter Cove, King George Island. Ber. Polarforsch., 299: 113-118.

Thatje, S. and E. Mutschke. - 1999. Distribution of abundance, biomass, production and productivity of macrobenthos in the Subantarctic Magellan Province (South America). Polar Biol., 22: $31-37$.
Vera, P., S. Avaria and P. Muñoz. - 1996. Composición y distribución del fitoplancton de los fiordos adyacentes a Campo de Hielo Sur y su relación con algunos parámetros oceanográficos. Cienc. Tecnol. Mar., 19: 73-92.

Wloddarska-Kowalczuk, M. and T.H. Pearson. - 2004. Soft-bottom macrobenthic faunal associations and factors affecting species distributions in an Arctic glacial fjord (Kongsfjord, Spitsbergen). Polar Biol., 27: 155-167.

Wloddarska-Kowalczuk, M., J.M. Wesawski and L. Kotwicki. 1998. Spitsbergen glacial bays macrobenthos - a comparative study. Polar Biol., 20: 66-73. 